\title{
Genetic dissection of the redundant and divergent functions of histone chaperone paralogs in yeast
}

19 Neda Savic ${ }^{1}$, Shawn P. Shortill ${ }^{1}$, Misha Bilenky², David Dilworth ${ }^{1}$, Martin Hirst ${ }^{2}$, Christopher J. 20 Nelson $^{1}$

$22{ }^{1}$ Dept. Biochemistry and Microbiology, University of Victoria, Victoria, BC, V8W 3P6, Canada.

$23 \quad{ }^{2}$ BC Cancer Agency Genome Sciences Centre and the Department of Microbiology \& Immunology, 24 Michael Smith Laboratories, University of British Columbia, Vancouver, BC, V6T 1Z3, Canada

* Correspondence: cjn@uvic.ca

32 Running title: Fpr3 and Fpr4 have overlapping and divergent functions 
35 Gene duplications increase organismal robustness by providing freedom for gene divergence or 36 by increasing gene dosage. The yeast histone chaperones Fpr3 and Fpr4 are paralogs that can assemble 37 nucleosomes in vitro, however the genomic locations they target and their functional relationship is 38 poorly understood. We refined the yeast synthetic genetic array (SGA) approach to enable the functional 39 dissection of gene paralogs. Applying this method to Fpr3 and Fpr4 uncovered their redundant and 40 divergent functions: while Fpr3 is uniquely involved in chromosome segregation, Fpr3 and Fpr4 co41 operate on some genes and are redundant on others where they impact gene expression and transcriptional 42 processivity. We find that the TRAMP5 RNA exosome is essential in $\Delta f p r 3 \Delta f p r 4$ yeast and leverage this 43 information to identify Fpr3/4 target loci. Amongst these are the non-transcribed spacers of ribosomal 44 DNA where either paralog is sufficient to establish chromatin that is both transcriptionally silent and 45 refractory to recombination. These data provide evidence that Fpr3 and Fpr4 have shared chromatin46 centric functions, especially at nucleolar rDNA. However, their distinct genetic interaction profiles show 47 they also have evolved separate functions outside of the nucleolus.

53 Keywords: chromatin/ functional divergence/ FKBPs/ genetic interactions/ nucleolus 


\section{Introduction}

Gene duplication events play an important role both in driving protein evolution and in providing a mechanism for ensuring the robustness of biological systems. Since the earliest observations of duplications on chromosomes (Darlington \& Moffett, 1930; Bridges, 1936) and redundant genes (Kataoka et al, 1984; Basson et al, 1986), models implicating gene duplication events as complex drivers of evolution have been proposed (Ohno, 1970; Hughes, 1994; Force et al, 1999; Francino, 2005; Innan \& Kondrashov, 2010). Evolutionary forces can favor the retention of redundant genes for dosage reasons, for example, identical copies of histone and ribosomal genes are present in most eukaryotes. Alternately, duplicated genes provide an opportunity for functional divergence of gene pairs, or paralogs, over time.

FPR3 and FPR4 are two S. cerevisiae paralogs (Manning-Krieg et al, 1994; Shan et al, 1994; Benton et al, 1994; Dolinski et al, 1997) derived from a distant whole genome duplication event (Pemberton, 2006; Wolfe \& Shields, 1997; Kellis et al, 2004). They code for highly similar proteins (58\% identical and $72 \%$ similar in amino acid residues) with acidic N-terminal nucleoplasmin-like histone chaperone and C-terminal FK506-binding (FKBP) peptidyl-prolyl isomerase domains (Kuzuhara \& Horikoshi, 2004; Xiao et al, 2006; Park et al, 2014) (Figure 1 A). Both proteins localize to the nucleus and are enriched in the nucleolus (Manning-Krieg et al, 1994; Shan et al, 1994; Benton et al, 1994; Huh et al, 2003). Notably, Fpr3 and Fpr4 interact with each other and share some common physical interactors (Krogan et al, 2006), including histones (Shan et al, 1994; Xiao et al, 2006; Nelson et al, 2006), and the Nop54 ribosome biogenesis factor (Sydorskyy et al, 2005). Additionally, both Fpr3 and Fpr4 are multicopy suppressors of temperature sensitivity and mating defects resulting from the absence of the Tom 1 E3 ubiquitin ligase (Davey et al, 2000; Utsugi et al, 1999). Therefore, there is good evidence that Fpr3 and Fpr4 operate together and may have redundant functions.

There is also evidence that these enzymes are not equivalent. Fpr3 has been identified as a regulator of chromosome dynamics at mitotic and meiotic centromeres. During meiosis, Fpr3 enhances recombination checkpoint delay (Hochwagen et al, 2005) and prevents meiotic chromosome synapsis initiation at centromeres (Macqueen \& Roeder, 2009). Fpr3 is also required for the degradation of the centromeric histone $\mathrm{H} 3$ variant Cse4 (Ohkuni et al, 2014). To our knowledge, no reports describe similar data for Fpr4. Taken together, these reports are evidence that Fpr3 and Fpr4 may have functionally diverged.

The comparative impact(s) of Fpr3 and Fpr4 in gene expression are also unclear. While Fpr4 can silence expression of a reporter at ribosomal DNA (rDNA) (Kuzuhara \& Horikoshi, 2004) and is involved in transcription induction kinetics through the isomerization of prolines on the amino tails of 
histones H3 and H4 (Nelson et al, 2006), the degree to which Fpr3 regulates transcription has not been described.

In yeast, the loss-of-function phenotypes and genetic interactions of chromatin regulators usually provide insight to their chromatin-centric functions. For example, the yeast histone chaperone coding genes ASF1 and RTT106 display clear chromatin-related genetic interactions in synthetic genetic array (SGA) screens (Costanzo et al, 2010, 2016). We noted that the genetic interactomes of FPR3 and FPR4 contained few chromatin-related hits (Costanzo et al, 2010, 2016; Collins et al, 2007; Stirling et al, 2011; Milliman et al, 2012) and hypothesized that the high similarity of these paralogs renders them semiredundant, masking their genetic interactions.

Here, through a set of comprehensive genetic interaction screens designed for paralogs, we reveal that the functions of Fpr3 and Fpr4 are complex, and include separate, co-operative and redundant functions in chromatin and chromosome biology. Deletion of $\Delta t r f 5$, a key component of the TRAMP5 RNA exosome renders cells reliant on Fpr3/4 for viability, transcriptional processivity and silencing. This strongly suggests that Fpr3/4 and TRAMP5 regulate common RNA transcripts through RNA degradation and chromatin-mediated silencing, respectively. Finally, a major chromatin target for these chaperones is found within the nucleolar rDNA where either protein is sufficient to promote both silencing and genomic stability at the non-transcribed spacer regions. Taken together we have developed paralogs. Applying this to Fpr3/4 has revealed that these histone chaperones have a redundant ancestral function in chromatin regulation of rDNA, however, we also provide evidence that they co-operate and are in the process of functionally diverging.

\section{Results}

\section{Genetic interactions reveal separate, co-operative, and redundant functions of FPR3 and FPR4}

114 masking genetic interactions. To address this and determine the biological processes sensitive to these 115 histone chaperones we performed a modified synthetic genetic array (SGA) screen designed to dissect 116 functional redundancy of gene paralogs (Figure $1 \mathrm{~B}$, see materials and methods). To this end we crossed 117 a dual-query $\Delta f p r 3 \Delta f p r 4$ double mutant strain to the 4784 strain non-essential yeast deletion mutant array 118 (DMA), so that the fitness of all double ( $\Delta$ fpr3 $\Delta x x x$ and $\Delta f p r 4 \Delta x x x)$ and triple ( $\Delta$ fpr $3 \Delta f p r 4 \Delta x x x)$ mutant 119 meiotic progeny could be measured. The query strain also harbored an episomal URA3 plasmid with a 
121 vulnerability to suppressor mutations. This plasmid was maintained until the final step of the screen when 122 counter-selection with 5'FOA created the fpr4 null status. Using standard selection methods, the spores 123 of this single cross were manipulated to generate three separate SGA screens that identified all synthetic 124 lethal/sick interactions with $\Delta f p r 3$, with $\Delta f p r 4$ and genes whose disruption only exacerbated fitness of 125 yeast lacking both $\Delta f p r 3 \Delta f p r 4$.

126 We identified 456 and 138 genetic interactors that were unique to either FPR3 or FPR4, respectively, 127 revealing that these paralogs are not equivalent (Figure $1 \mathrm{C}$ top). However, 78 genes interacted with both 128 FPR3 and FPR4, implying that there are specific contexts of paralog co-operativity, that is situations 129 where both histone chaperone is required for function. We also uncovered 75 masked interactors, defined 130 as genes whose deletion only impacts the fitness $\Delta f p r 3 \Delta f p r 4$ yeast (Figure $1 \mathrm{C}$ bottom). These genes 131 highlight processes when paralog function is redundant. The complete list of these genes and a gene 132 ontology analysis are provided in Appendix file 1 and Appendix file 2 respectively.

133 FPR3 genetic interactors include members of the large and small mitochondrial ribosomal subunits $134\left(\mathrm{P}=3.42 \times 10^{-11}\right.$ and $\mathrm{P}=8.38 \times 10^{-7}$ respectively), the mitochondrial pyruvate dehydrogenase complex $135\left(\mathrm{P}=6.48 \times 10^{-4}\right)$, the cytochrome bc1 complex $\left(\mathrm{P}=1.49 \times 10^{-3}\right)$ and components of the ESCRT II endosomal 136 sorting complex $\left(\mathrm{P}=8.85 \times 10^{-3}\right)$ (Figure $\left.1 \mathrm{D}\right)$. We also identified all three components of the Ctk1 kinase 137 complex $\left(\mathrm{P}=1.69 \times 10^{-4}\right)$, and four components of the $\operatorname{Swr} 1$ chromatin remodeler $\left(\mathrm{P}=4.45 \times 10^{-3}\right)$ supporting 138 at least some potential chromatin centric roles of Fpr3. Most notably, we uncovered complexes involved 139 in chromosome segregation such as the astral microtubule $\left(\mathrm{P}=2.03 \times 10^{-6}\right)$, kinetochore $\left(\mathrm{P}=2.38 \times 10^{-4}\right)$, and 140 the Mrc1/Csm3/Tof1 complex $\left(\mathrm{P}=1.69 \times 10^{-4}\right)$ as genetic interactors unique to Fpr3, and not Fpr4. These 141 systems-level data support reports which indicate that Fpr3, but not Fpr4, regulates mitotic and meiotic 142 chromosome dynamics, including those associated with centromeres (Hochwagen et al, 2005; Macqueen 143 \& Roeder, 2009; Ohkuni et al, 2014). Although we identified 138 FPR4 specific genetic interactions, 144 they fall into limited ontologically related categories. Several genes coding for components of the pre145 autophagosome and associated with the process of mitochondrial degradation $\left(\mathrm{P}=1.29 \times 10^{-3}\right)$ were the 146 exception, but the relationship between Fpr4 and these processes is not clear. Taken together the number 147 and nature of the genetic interactions from single query screens suggest that Fpr4 cannot fulfil many of 148 Fpr3's biological functions, particularly those in chromosome dynamics, and mitochondrial ribosome 149 biology. However, Fpr3 might be competent to substitute for Fpr4 (see below).

150 Shared genetic interactions would be expected if both paralogs were required for the efficient 151 execution of a biological process. Among genetic interactors common to both FPR3 and FPR4 are genes 152 coding for the ESCRT III complex $\left(\mathrm{P}=1.44 \times 10^{-6}\right)$ which functions in endosomal sorting, the 153 Ada2/Gcn5/Ada3 histone acetyltransferase $\left(\mathrm{P}=3.57 \times 10^{-6}\right)$ and the ATP-dependent SWI/SNF chromatin 
remodeler (Figure 1 D). Shared genetic interactions with the SWI/SNF remodeler were confirmed using spotting assays (data not shown). The proposed co-operation of Fpr3 and Fpr4 is supported by the fact these proteins co-purify (Krogan et al, 2006) and, like nucleoplasmin, have the intrinsic propensity to form oligomers (Edlich-Muth et al, 2015; Dutta et al, 2001; Koztowska et al, 2017). Thus, these shared genetic interactions with known chromatin regulatory complexes support published protein complex data and indicate that Fpr3 and Fpr4 likely co-operate through heteromeric complexes in some contexts.

75 masked genetic interactions are only detectible in double $\Delta f p r 3 \Delta f p r 4$ mutants (Figure $1 \mathrm{C}$ bottom). These genes are essential only when both paralogs are absent, and thus highlight processes in which Fpr3 and Fpr4 are redundant. Most notably, these interactors include three non-essential components of the TRAMP5 nuclear RNA exosome (TRF5, AIR1, and RRP6) (Figure $2 \mathrm{~A}$ ), an RNA surveillance factor that recognizes, polyadenylates and degrades aberrant RNA transcripts (Figure 2 B) (LaCava et al, 2005;

synthetic sickness of $\Delta f p r 3 \Delta f p r 4$ with $\Delta r r p 6$ and $\Delta t r f 5$ using growth curves (Figure $2 \mathrm{C}$ ). This demonstrates that Fpr3 and Fpr4 have redundant biological functions likely involving the negative regulation of RNAs.

\section{Suppressor genetic interactions of FPR3 and FPR4}

The SWI/SNF and Ada2/Gcn5/Ada3 complexes are particularly important for the fitness of $\Delta$ fpr3 and $\Delta f p r 4$ yeast (Figure $1 \mathrm{D})$. In support of a chromatin defect underlying these phenotypes, we found that several genetic suppressors (Figure 3), which alleviate the slow growth phenotype of $\Delta$ fpr $3 \Delta f p r 4$ yeast, are themselves chromatin modifiers including: three NAD+ dependent histone deacetylases $(\mathrm{P}=$ $\left.6.33 \times 10^{-5}\right)$, Hos2, Hdal and Hos3; three of the four components of the HIR replication-independent nucleosome assembly complex $\left(\mathrm{P}=1.29 \times 10^{-5}\right)$, Hir1, Hpc2, and Hir3; and Swd3 and Sdc1, two of the eight components of the Set1/COMPASS histone H3K4 methylase complex, $\left(\mathrm{P}=5.87 \times 10^{-3}\right)$. We note that the Swd2 subunit of COMPASS is encoded by an essential gene and the Aset1 knockout is not present in our deletion strain collection. It is particularly notable that we find histone deacetylases enriched among suppressor interactions and histone acetyltransferases among synthetic sick and lethal interactions. The presence of both aggravating and alleviating chromatin-related genetic interactions in our modified SGA screen is consistent with a chromatin-centric mode of action for Fpr3 and Fpr4.

\section{Fpr3 and Fpr4 have shared and separate transcriptional targets}

The genetic interactions of Fpr3 and Fpr4 with known chromatin modifiers suggest that they regulate transcription. Consistent with this, Fpr4 directly represses transcription from a reporter gene both in an 
artificial recruitment assay (Park et al, 2014) and integrated in the rDNA repeats of yeast (Kuzuhara \& Horikoshi, 2004). Fpr4 is also bound to multiple genomic locations (Nelson et al, 2006; Kuzuhara \& Horikoshi, 2004). To determine the impact of these proteins on transcription genome-wide, we sequenced the ribo-minus fraction of RNAs from wt, $\Delta f p r 3, \Delta f p r 4$ and $\Delta f p r 3 \Delta f p r 4$ yeast (Appendix file 3, and Figure $4 \mathrm{~A}$ ). We included a $\Delta$ sir2 strain as a control, which in our analysis displays 854 differentially expressed genes using a lenient cut-off of 1.3 fold. This number and nature of Sir2regulated genes is in good agreement with previous reports of Sir2 regulated genes and binding sites (Ellahi et al, 2015; Li et al, 2013).

Single deletion mutants of $\Delta f p r 3$ and $\Delta f p r 4$ had 524 and 549 differentially expressed genes, respectively (Appendix file 3). Surprisingly, double $\Delta f p r 3 \Delta f p r 4$ mutants did not exhibit a major additive effect with only 683 differentially regulated genes. In each of the three above experiments, approximately $1 / 3$ of differentially expressed genes were upregulated. These genes represent transcripts repressed by the histone chaperone(s) and include members of the cytosolic large ribosomal subunit $\left(\mathrm{P}=3.40 \times 10^{-11}\right.$ in $\Delta f p r 3$ mutants, $\mathrm{P}=8.94 \times 10^{-8}$ in $\Delta f p r 4$ mutants, and $\mathrm{P}=4.41 \mathrm{X} 10^{-12}$ in $\Delta f p r 3 \Delta f p r 4$ mutants), components of the cytosolic small ribosomal subunit $\left(\mathrm{P}=8.99 \times 10^{-6}\right.$ in $\Delta f p r 3$ mutants, $\mathrm{P}=5.84 \times 10^{-6}$ in $\Delta f p r 4$ mutants, and $2.69 \times 10^{-10}$ in $\Delta f p r 3 \Delta f p r 4$ mutants) and components of the fungaltype cell wall $\left(\mathrm{P}=1.47 \times 10^{-4}\right.$ in $\Delta f p r 3$ mutants, $\mathrm{P}=4.90 \times 10^{-4}$ in $\Delta f p r 4$ mutants, and $\mathrm{P}=2.56 \times 10^{-3}$ in $\Delta f p r 3 \Delta f p r 4$ mutants). Some of the most differentially expressed genes (up to 60 fold) include proteins involved in phosphate metabolic processes such as the PHO5 and PHO11/12 acid phosphatases, and the phosphate transporters $\mathrm{PHO} 89$, $\mathrm{PHO} 84$ and $\mathrm{PIC} 2\left(\mathrm{P}=9.77 \times 10^{-7}\right.$ in $\Delta$ fpr3 mutants, $\mathrm{P}=3.51 \mathrm{X} 10^{-5}$ in $\Delta$ fpr 4 mutants, and $\mathrm{P}=1.77 \mathrm{X} 10^{-4}$ in $\Delta f p r 3 \Delta f p r 4$ mutants, Appendix file 4).

Two-thirds of differentially regulated genes are positively regulated by Fpr3/4. These include fungal type cell wall organization factors $\left(\mathrm{P}=1.36 \times 10^{-5}\right.$ in $\Delta$ fpr3 mutants, $\mathrm{P}=2.14 \times 10^{-3}$ in $\Delta f p r 4$ mutants, and $\mathrm{P}=1.62 \times 10^{-3}$ in $\Delta f p r 3 \Delta f p r 4$ mutants $)$; proteins involved in iron ion homeostasis $\left(\mathrm{P}=1.91 \times 10^{-7}\right.$ in $\Delta f p r 3$ mutants, $\mathrm{P}=2.53 \times 10^{-5}$ in $\Delta f p r 4$ mutants, and $\mathrm{P}=9.86 \times 10^{-5}$ in $\Delta$ fpr $3 \Delta f p r 4$ mutants); and pheromone response, mating type determination and sex specific proteins $\left(\mathrm{P}=3.26 \times 10^{-8}\right.$ in $\Delta$ fpr 3 mutants, $\mathrm{P}=2.42 \times 10^{-4}$ in $\Delta f p r 4$ mutants, and $\mathrm{P}=2.20 \times 10^{-5}$ in $\Delta f p r 3 \Delta f p r 4$ mutants).

Since roughly one third of Fpr3 regulated transcripts are also regulated by Fpr4, and vice versa, we conclude that, on these genes, transcriptional regulation requires both Fpr3 and Fpr4. In other words, Fpr3 and Fpr4 co-operate in these contexts. Like the SWI/SNF complex, the impact of the Fpr3 and Fpr4 


\section{The TRAMP5 RNA exosome masks the impact of Fpr3/4}

Considering that the TRAMP5 nuclear RNA exosome is essential in yeast lacking Fpr3 and Fpr4, we wondered whether this RNase could be masking changes in the $\Delta f p r 3 \Delta f p r 4$ transcriptome. To test this idea, we sequenced RNA from isogenic $\Delta \operatorname{trf5}$ deficient yeast from our SGA screen, comparing those with

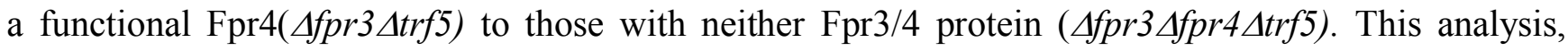
designed to reveal Fpr4 regulated RNAs, uncovered a total of 1321 differentially expressed genes (967 upregulated and 354 downregulated) (Figure $4 \mathrm{~B}$ ). The increase in upregulated transcripts in this experiment supports the hypothesis that Fpr4 negatively regulates a breadth of genes, and that these

RNAs are also substrates for the TRAMP5 RNA exosome. As expected, upregulated genes coding for protein components of the cytosolic ribosome $\left(\mathrm{P}=3.21 \mathrm{X}^{-12}\right)$ (including the cytosolic large ribosomal subunit $\mathrm{P}=3.00 \times 10^{-7}$ and the cytosolic small ribosomal subunit $\mathrm{P}=9.48 \times 10^{-4}$ ) and genes associated with rRNA processing $\left(\mathrm{P}=1.14 \times 10^{-8}\right)$ are highly enriched as Fpr4 targets. Also enriched were genes coding for constituents of the fungal-type cell wall $\left(\mathrm{P}=1.87 \mathrm{X} 10^{-4}\right)$ and the electron transport chain $\left(6.12 \mathrm{X} 10^{-8}\right)$ (Figure $4 \mathrm{C}$ ). Taken together the ontologies associated with upregulated transcripts in $\Delta f p r 3 \Delta f p r 4 \Delta t r f 5$ triple mutants indicate that Fpr3 and Fpr4 negatively regulate discreet subsets of genes, particularly those involved in ribosome biogenesis. That Fpr3/4 and TRAMP5 negatively regulate overlapping transcripts provides a potential explanation for their synthetic lethality.

\section{A signature of abortive transcription in $\Delta f p r 3 \Delta f p r 4$ yeast}

Further interrogation of the transcriptome data reveals evidence for Fpr3 and Fpr4 in transcriptional processivity: approximately $40 \%$ of differentially expressed genes in $\Delta f p r 3 \Delta f p r 4 \Delta t r f 5$ yeast displayed an accumulation of reads towards the 5' end of the annotated transcript. Subsequent bioinformatic analysis of the total transcriptomes of $\Delta f p r 3 \Delta f p r 4 \Delta t r f 5$ and $\Delta f p r 3 \Delta \operatorname{trf5}$ mutants revealed that this 5 '-biased asymmetry is widespread, and detectable in genes irrespective of their net change in transcription (Figure 5 A). Two example genes illustrating this asymmetry signature are presented in Figure 5 B; SSF1 codes for a constituent of the $66 \mathrm{~S}$ pre-ribosome and is required for large ribosomal subunit maturation, while UTP1 codes for a component required for proper endonucleolytic cleavage of $35 \mathrm{~S}$ rRNA. The pairedend tag coverage associated with both of these genes, but not the IDPI gene (Figure $5 \mathrm{C}$ ), displays the characteristic 5'assymetry in $\Delta f p r 3 \Delta f p r 4 \Delta t r f 5$ yeast. This signature demonstrates Fpr3 and Fpr4 negatively regulate transcription from many promoters and suggests that in the absence of these histone chaperones, transcription can initiate, but may not proceed to completion. That these abortive mRNAs 


\section{Fpr3 and Fpr4 inhibit transcription from the non-transcribed spacers of ribosomal DNA}

The ribosomal DNA locus in yeast consists of a series of 150-200 tandem repeats of a $9.1 \mathrm{~kb}$ unit containing the $35 \mathrm{~S}$ and the $5 \mathrm{~S}$ rRNAs each separated by two non-transcribed spacer sequences (NTS1 and NTS2) (Figure 6 A top) (Johnston et al, 1997). Given the nucleolar enrichment of Fpr3 and Fpr4, and the ability of Fpr4 to repress reporter expression from rDNA (Kuzuhara \& Horikoshi, 2004), we asked if yeast lacking Fpr3 and Fpr4 also display transcriptional defects at rDNA. While our RNA-seq analysis was performed on ribo-minus RNA, reads from rRNA are readily detected (presumably from incomplete rRNA depletion) and indicated no change in rRNA production, which we have also observed in Northern and qRT-PCR analyses (data not shown). However, disruption of both Fpr3 and Fpr4 has a profound impact on silencing at NTS1 and NTS2 (Figure 6 A). Transcripts from both strands of NTS1 and NTS2 accumulate in $\Delta f p r 3 \Delta f p r 4 \Delta t r f 5$ strains. Consistent with previous reports (Kuzuhara \& Horikoshi, 2004), we also find that the repression of a URA3 reporter gene integrated at the NTS1 region of rDNA requires Fpr3 and Fpr4 (Figure 6 B). Taken together, these results support a model where Fpr3 and Fpr4 establish a transcriptionally silent chromatin structure at rDNA.

\section{Fpr3 and Fpr4 contribute to genomic stability at ribosomal DNA}

Ribosomal RNAs comprise approximately $80 \%$ of the total RNA in yeast; accordingly the $\sim 50 \%$ of rDNA tandem repeats that are transcribed in a given cell are the most heavily transcribed, and nucleosome-free, genes in the cell (Nomura et al, 2004; Warner, 1999; Vogelauer et al, 2000). Reciprocally, the adjacent rDNA non-transcribed spacers (NTS) and inactive rDNA repeats are chromatinized and potently silenced. This arrangement is thought to generate a chromatin template that is refractory to recombination between rDNA repeats and the deleterious loss of rDNAs from chromosome 12, which is a major driver of yeast replicative aging (Sinclair \& Guarente, 1997). For this reason, failure to generate heterochromatin environments at rDNA, as occurs in $\Delta$ sir2 histone deacetylase mutants, decreases genomic stability at this locus (Gottlieb \& Esposito, 1989; Kobayashi et al, 2004). We reasoned that if Fpr3 or Fpr4 were silencing the NTS regions via a mechanism that involves chromatin structure, that yeast lacking these enzymes should also exhibit genomic instability at this locus. To test this hypothesis, we introduced $\Delta f p r 3 \Delta f p r 4$ and $\Delta$ sir2 deletions into a strain with a reporter gene (URA3) integrated at NTS1 (Van Leeuwen et al, 2002; van Leeuwen \& Gottschling, 2002). First, URA+ status of each strain was confirmed, followed by growth in non-selective media (YPD) for two days to 
URA3 gene loss via recombination (Figure 7 A top). To discriminate between these events, we replica plated these individual isolates to media lacking uracil, where growth would indicate that the $U R A 3$ phenotype was a consequence of epigenetic silencing. Reciprocally, isolates that failed to grow would represent reporter loss events (Figure 7 A). These propagation assays revealed that normally, the rate of epigenetic switching of URA3 is much higher than reporter loss: $82 \%$ of $u r a$ - isolates still have a URA3 gene at the end of our propagation assay as exemplified growth in the absence of uracil (Figure $7 \mathrm{~B}$ and C), and by PCR of genomic DNA (not shown). As expected, Asir2 yeast are unable to establish silent chromatin at NTS1, and can only grow on 5'FOA via loss of the reporter. Finally, we observe that $\Delta f p r 3 \Delta f p r 4$ yeast are compromised in their ability to silence $U R A 3$ epigenetically: only $30 \%$ of 5 'FOA resistant colonies retain the $U R A 3$ gene. Thus, in $\Delta f p r 3 \Delta f p r 4$ yeast recombination and URA3 reporter gene loss are more frequent than epigenetic silencing. This observation supports a model where Fpr3 and Fpr4 build chromatin structures at the NTS regions of rDNA locus. These structures are critical to maintaining genome stability at rDNA.

\section{Discussion}

Gene duplication events play a critical role in protein and organism evolution. However, the high similarity of duplicated genes can lead to complete or partial compensation when one paralog is deleted, as is in the case in conventional genetic interaction analysis. Here we present a dual-query SGA screening approach where one genetic cross can report the separate and redundant genetic interactions of each paralog. Using this approach on two nucleoplasmin-like histone chaperones revealed that they perform separate, cooperative, and redundant chromatin-related functions. Given that approximately $13 \%$ of yeast protein coding genes are duplicates (Wolfe \& Shields, 1997), this approach may have wide applications to other studies of yeast paralogs.

The genetic interactions annotated here support a unique function for Fpr3 in orchestrating centromeric chromatin dynamics during chromosome segregation. This is fully consistent with existing literature (Hochwagen et al, 2005; Macqueen \& Roeder, 2009; Ghosh \& Cannon, 2013; Krogan et al, 2006; Ohkuni et al, 2014). Our comparative analyses provide additional systems-level evidence that this role is not shared with Fpr4 indicting that Fpr3, potentially as homo-oligomers, may regulate chromatin in a way that impacts chromosome segregation (Macqueen \& Roeder, 2009; Hochwagen et al, 2005). Furthermore, the fact that $\Delta f p r 3 \Delta f p r 4$ double mutants display fewer genetic interactions than single gene $\Delta f p r 3$ mutants (Appendix file 1) indicates that Fpr4 may be toxic in the absence of Fpr3 (Ohkuni et al, 2014). This model predicts that, in the absence of Fpr3, the partial engagement or modification of chromatin by Fpr4 is deleterious. 
SWI/SNF complex members are shared interactors of Fpr3 and Fpr4, appearing as hits in both single mutant screens. These results could be explained by reduced dosage of a histone chaperone. Alternately, these genetic interactions are consistent with a model where Fpr3 and Fpr4 act together to chaperone nucleosomes, facilitating chromatin dynamics as SWI/SNF does. Whether this means that the chaperones operate together in a sequence of events, such as the removal and subsequent redeposition of nucleosomes during transcription or, in concert as a hetero-oligomeric complex, is not yet clear. The fact that Fpr3 and Fpr4 co-purify (Krogan et al, 2006) supports the latter model, but does not exclude the former.

The repression of several PHO genes in rich media requires both Fpr3 and Fpr4. The PHO5, PHO11/12 acid phosphatases, and the PHO89, PHO84 and PIC2 phosphate transporters are intimately linked to the metabolism of both phosphate and intracellular polyphosphate stores. It is therefore intriguing that both Fpr3 and Fpr4 were recently identified as two of the major polyphosphorylated proteins in yeast along with several proteins in an evolutionarily conserved network of ribosome biogenesis factors (Bentley-DeSousa et al, 2018). The precise sites of Fpr3/4 polyphosphorylation and their impact on function is not yet clear. However, the identification of the well-studied PHO5 gene as an Fpr3 and Fpr4 target provides an ideal system for determining the impact of this new post-translational modification on these histone chaperones.

The yeast TRAMP5 complex recognizes and polyadenylates aberrant RNA transcripts in order to target them for degradation by the Rrp6 ribonuclease (Karyn Schmidt and J. Scott Butler, 2013). TRAMP5 targets include both ribosomal protein coding mRNAs and cryptic unstable transcripts generated from intragenic sites on the genome including those within the ribosomal DNA locus (Reis \& Campbell, 2007; San Paolo et al, 2009; Wery et al, 2009; LaCava et al, 2005). Here we found that deletion of $\Delta \operatorname{trf} 5$ enabled the detection of an unexpected transcriptome signature in $\Delta f p r 3 \Delta f p r 4$ yeast where there is a bias in RNA-seq reads towards the 5' end of genes. This means that Fpr3/4 redundantly promote the transcriptional elongation process. It is noteworthy that these reads appear to cover the first 1-3 nucleosomes of genes because Fpr3/4 have evolved basic surface features to permit nucleosome binding (Leung et al, 2017) and that Fpr4 was previously shown to be important for the kinetics of transcriptional induction (Nelson et al, 2006). Thus, the nucleosomes immediately downstream of the transcriptional start site are candidates targets of Fpr3/4. This regulation could involve either depositing histones within promoters to inhibit transcriptional initiation or the eviction of nucleosomes from sequences downstream of the promoter in order to remove nucleosome blocks to the polymerase. These models are currently under investigation.

Fpr3/4 have the greatest impact on the steady-state levels of mRNAs encoding ribosomal protein genes and rRNA processing machinery. Thus, Fpr3/4 may function as master regulators of ribosome 
biogenesis by coordinating both ribosomal protein abundance and rRNA processing. Given that many ribosomal and rRNA processing protein genes are driven by common regulators, Fpr3/4 may recognize common DNA sequences or transcription factors to accomplish this function (Fermi et al, 2016). It appears that at least some elements of this ribosomal biogenesis function are conserved in the human nuclear FKBP25 protein (Gudavicius et al, 2014; Dilworth et al, 2017).

In addition to regulating the transcription of protein coding genes Fpr3 and Fpr4 restrict transcription from the non-transcribed spacers (NTS) sequences of ribosomal DNA. This is consistent with both their nucleolar enrichment and data indicating that they inhibit transcription of exogenous reporters at NTS2 in yeast (Kuzuhara \& Horikoshi, 2004) and endogenous 18S rDNA in plants (Li \& Luan, 2010). In yeast the NTS loci contain important DNA sequence features including as two terminators for the RNA PolI transcribed RDN35 repeat, a replication fork barrier site, and an autonomous replication site. Two separate observations suggest that Fpr3 and Fpr4 function redundantly to build chromatin at rDNA in order to insulate DNA at these spacers. First, yeast lacking both paralogs accumulate large amounts of aberrant NTS RNA transcripts, and these RNAs are templated by both DNA strands. Second, consistent with a chromatin structure defect underpinning this phenomenon, we find that the rDNA locus in $\Delta f p r 3 \Delta f p r 4$ yeast is also hyper-recombinogenic (Figure 6). Thus, Fpr3 and Fpr4 are histone chaperones of particular importance at the 100-200 rRNA repeats where they mediate the stability and silencing of chromatin structure at this locus, and how the structure differs from other targets in the nuclear genome remain open questions.

\section{Materials \& Methods}

\section{Yeast strains and plasmids}

Yeast strains used in this study are described in Appendix file 5. Strains in the MAT a non-essential yeast deletion collection (DMA) used for the SGA analysis are all isogenic to BY4741 and were purchased from Thermofisher Dharmacon. The plasmid rescued double genomic deletion $\Delta f p r 3 \Delta f p r 4$ SGA query strain (YNS 35) was created in a Y7092 genetic background as follows. The endogenous FPR4 locus on a Y7092 wt strain was replaced with a nourseothricin resistance (MX4-NATR) PCR product deletion module. The resulting single gene $\Delta f p r 4$ deletion mutant was subsequently transformed with prs316 FPR4: a single copy URA3 marked shuttle vector carrying an untagged full length copy of the FPR4 open reading frame with endogenous promoter and terminator (originally described in (Nelson 
et al, 2006)). The endogenous FPR3 locus on this plasmid rescued $\triangle f p r 4$ deletion mutant was subsequently replaced with a $L E U 2$ PCR product deletion module.

Triple deletion mutants: $\Delta r r p 6 \Delta f p r 3 \Delta f p r 4$ and $\Delta \operatorname{trf5} \Delta f p r 3 \Delta f p r 4$ and their corresponding mixed population total haploid meiotic progeny controls used in the validating growth curves were generated from the SGA cross (see below).

Single gene deletion mutants of $\Delta f p r 3, \Delta f p r 4$, and $\Delta$ sir2 used for the RNA sequencing are all isogenic to BY4741 and were either purchased from open biosystems, or taken from the yeast deletion collection (purchased from Thermofisher Dharmacon). The isogenic double deletion $\Delta f p r 3 \Delta f p r 4$ mutant was constructed from the open biosystems $\Delta f p r 3$ single gene deletion mutant by replacing the endogenous FPR4 locus with a nourseothricin resistance $(M X 4-N A T R)$ PCR product deletion module. The

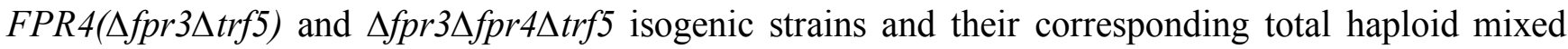
population controls were generated from the SGA cross (see below).

The $\Delta f p r 3$ and $\Delta f p r 4$ deletion mutant strains used in the rDNA reporter spotting assays were generated from a cross of the MAT $\alpha$ UCC1188 (Van Leeuwen et al, 2002) with a MATa BY4741 deletion mutant see Appendix table 5 for details. The UCC1188 background $\Delta f p r 3 \Delta f p r 4$ double deletion mutant, UCC1188 background $\Delta$ sir2 deletion mutant, and HML $\alpha$ reporter expression mutants were generated by lithium acetate transformation of either UCC1188 or UCC7266 (Van Leeuwen et al, 2002) with PCR product deletion modules. The $\Delta f p r 3 \Delta f p r 4$ and $\Delta$ sir2 deletion mutant strains used in the propagation assays were generated from a transformation of UCC1188 with PCR product deletion modules.

\section{Synthetic Genetic Array (SGA) Analysis}

SGA analysis was performed using a Singer Instruments ROTOR microbial arraying robot as previously described (Tong \& Boone, 2006) with the following modifications. The MAT a/ $\alpha$ diploid zygotes resulting from the query strain DMA cross were pinned onto diploid selective YPD + G418/clonNAT plates a total of two times for greater selection against any residual haploids. Sporulation was carried out at room temperature for 14 days. Spores were pinned onto Mat a selective germination media for two rounds of selection as previously described (Tong \& Boone, 2006).

The resulting MAT a progeny were subsequently replica plated onto four kinds of selective media: control media selective for the total haploid meiotic progeny population (SD media lacking histidine, arginine, lysine and containing canavanine and thialysine both at a final concentration of 50mg/l, and G418 at a final concentration of $200 \mathrm{mg} / \mathrm{L}$ ), media selective for $\Delta x x x \Delta$ fpr3 haploid meiotic progeny (SD media lacking histidine, arginine, lysine, leucine, uracil, and containing canavanine and thialysine both 
416 at a final concentration of $50 \mathrm{mg} / 1, \mathrm{G} 418$ and clonNAT both at a final concentration of 200mg/L), media

417 selective for $\Delta x x x \Delta f p r 4$ haploid meiotic progeny (SD media lacking histidine, arginine, lysine, and 418 containing canavanine and thialysine both at a final concentration of 50mg/1, G418 and clonNAT both at 419 a final concentration of $200 \mathrm{mg} / \mathrm{L}$, and 5-fluoroorotic acid at a final concentration of $1000 \mathrm{mg} / \mathrm{L}$ ), and 420 finally media selective for $\Delta x x x \Delta f p r 3 \Delta f p r 4$ haploid meiotic progeny (SD media lacking histidine, 421 arginine, lysine, leucine, and containing canavanine and thialysine both at a final concentration of $42250 \mathrm{mg} / \mathrm{l}, \mathrm{G} 418$ and clonNAT both at a final concentration of $200 \mathrm{mg} / \mathrm{L}$, and 5-fluoroorotic acid at a final 423 concentration of $1000 \mathrm{mg} / \mathrm{L}$ ). Plates were incubated at $30^{\circ} \mathrm{C}$ for 24 hours and were then expanded into 424 triplicate and incubated for an additional 24 hours at $30^{\circ} \mathrm{C}$.

425 Images of each plate were scanned and subsequently processed using the Balony image analysis software package as previously described (Young \& Loewen, 2013). In brief, pixel area occupied by each colony was measured to determine colony size. Progeny fitness was then scored as follows. The ratio of each double $(\Delta x x x \Delta f p r 3, \Delta x x x \Delta f p r 4)$ and triple ( $\Delta x x x \Delta f p r 3 \Delta f p r 4)$ mutant colony size relative to its corresponding total haploid meiotic progeny control colony was determined. Ratio cutoff thresholds were estimated automatically by the software by extrapolating the central linear portion of the ratio distributions and finding the $y$-intercepts at either ends of the $x$-axis. Default ratio cutoff thresholds were used (a complete list of all genetic interactions generated from each dataset is presented in Appendix file 1).

\section{SGA Data Processing}

Specific, common and masked synthetic sick/lethal interactors were identified as follows. First, duplicate genes from the list of hits from each dataset were identified and removed. The synthetic sick/lethal hits from each of the three datasets were then compared to each other in order to identify unique and common genes in each list. We thus identified a list of interactors unique to the $x x x$ fpr3 meiotic progeny and a list of interactors unique the $x x x$ fpr 4 meiotic progeny. Hits present in both the $x x x$ fpr3 meiotic progeny and the $x x x$ fpr4 meiotic progeny were identified as common interactors of FPR3 and FPR4. Hits that only appear in the xxxfpr3fpr4 meiotic progeny were identified as masked genetic interactors. Unique, common and masked suppressor interactors were identified the same way.

The lists specific, common, and masked synthetic sick/lethal and suppressor genetic interactors were subsequently analyzed using the web based FunSpec bioinformatics tool (http://funspec.med.utoronto.ca/, Dec 2017). The analysis was performed using a p-value cutoff score of 0.01, and without Bonferroni-correction. A full list of the ontologies uncovered and their corresponding 
$448 \mathrm{p}$ values is presented in Appendix file 2. Networks illustrating the specific and common genetic 449 interactions were drawn using the Cytoscape software platform (http://www.cytoscape.org/).

\section{Growth Curves}

Growth curves to validate the synthetic sickness phenotypes were carried out as follows. Colonies generated from the SGA assay corresponding to each triple mutant of interest and its respective control colony were isolated and validated for correct genotype by PCR. Confirmed strain isolates were then resuspended in fresh YPD media, normalized to an $\mathrm{OD}_{600}$ of 0.2 and distributed into triplicate wells of a 24 well cell culture plate. Plates were subsequently grown for $16 \mathrm{~h}$ at $30^{\circ} \mathrm{C}$ in a shaking plate reader. Readings of $\mathrm{OD}_{600}$ were taken every 30 minutes.

\section{RNA-Seq Library Preparation and Sequencing}

Single colony isolates of each strain were grown to mid log phase in $50 \mathrm{ml}$ of liquid yeast extractpeptone- dextrose (YPD) media. Samples were then pelleted and washed once with sterile water before being flash frozen in liquid nitrogen and stored for 16 hours at $-80^{\circ} \mathrm{C}$. Samples were thawed on ice, and RNA was extracted using a phenol freeze based approach as previously described (Schmitt et al, 1990). The extracted RNA was subsequently treated with RNase- free DNase I (Thermo Fisher Scientific)

RNA samples were processed and sequenced at the BC Cancer agency Michael Smith Genome Sciences Centre following standard operating protocols. Briefly, total RNA samples were ribo-depleated using the Ribo-Zero Gold rRNA Removal Kit (Yeast) (Illumina) and analyzed on an Agilent 2100 Bioanalyzer using Agilent 6000 RNA Nano Kit (Agilent Technologies, Santa Clara, California). cDNA was generated using the Superscript Double-Stranded cDNA Synthesis kit (ThermoFisher), 100bp paired-end libraries prepared using the Paired-End Sample Prep Kit (Illumina, San Diego, California).

\section{Processing of Sequencing Data}

Sequenced paired-end reads were aligned to the sacCer3 reference genome (https://www.ncbi.nlm.nih.gov/assembly/GCF_000146045.2/) using the BWA aligner (Li \& Durbin, 2010) (version 0.6.1-r104-tpx). We observed that out of 5110 Saccharomyces cerevisiae genes annotated in Ensembl v90 only 267 are spliced with and most of spliced genes (251) having one intron. Therefore, we considered genomic alignment of RNA-seq reads as a good approximation for the yeast transcriptome analysis. For every library total of $\sim 1.5-2 \mathrm{M}$ reads were sequenced, of which $\sim 75-95 \%$ of reads were aligned. 
To quantify gene expression, we filtered reads that aligned to multiple locations (and therefore can't be placed unambiguously) by applying a BWA mapping quality threshold of 5. We further collapsed fragments that were duplicated (only counting a single copy of a read pair if a number of pairs with the same coordinates was sequenced) as well removed chastity failed reads and considered only reads that were properly paired. Post-processing was performed using the 'pysam' application for python (https://github.com/pysam-developers/pysam). The alignment statistics were calculated using the 'sambamba' tool v 0.5.5 5 (Tarasov et al, 2015).

We considered cDNA fragment lengths distributions as well as genome-wide distributions of read coverage (data not shown) in order to ensure that these characteristics are similar for the pairs of data sets in the differential gene expression (DE) analysis. Genome wide pair-ended fragment coverage profiles for both strands were generated as well as read counts for every gene for further DE analysis.

The reads-per-kilobase-per-million (RPKM) values were calculated for every gene, and DE analysis was performed using the DEfine algorithm (M.Bilenky et al., unpublished). First, the chi2 p-value was estimated for every gene under the null hypothesis that the gene is not differentially expressed between two data sets. The Benjamini-Hochberg FDR-control procedure was applied (FDR=0.05) to find a $\mathrm{p}$ value threshold. To further reduce noise, we only considered genes with the fold-change (FC) between $\mathrm{RPKM}$ values $\mathrm{FC}>1.5$, as well required minimal number of aligned reads $>5$ per gene. Only reads aligned to the proper strand were considered in the DE analysis.

In addition to the standard DE analysis, where gene expression quantification was done by counting reads falling into the gene boundaries, we considered a model independent approach by calculating read counts in every 175bp long bin genome wide (for both strands), and performed DE analysis between bins (with the same approach we used for genes, see above). After defining the DE bins we overlapped their locations with gene coordinates to determine DE genes. This second approach also provided a list of potential DE expressed intergenic regions. A full list of the DE genes is presented in Appendix file 3.

\section{Ontology analysis of DE genes}

Ontologies associated with differentially expressed genes were identified using the web based FunSpec bioinformatics tool (http://funspec.med.utoronto.ca/, Dec 2018). The analysis was performed on genes displaying a fold change or 1.3 and up using a p-value cut-off score of 0.001 , and with Bonferroni-correction. A full list of the ontologies uncovered and their corresponding $\mathrm{p}$ values is presented in Appendix file 4. 
Universal gene coverage profiles were generated as follows; we first crated cDNA fragment coverage profiles genome wide for both strands using all aligned read-pairs. Next, we selected profiles for individual genes and scaled them to 100 units and normalized by the total gene coverage. After that we agglomerated all scaled and normalized gene coverage profiles together. When doing this, the profiles for genes on the negative strand were inverted (in other words we always agglomerate profiles from 5' to 3 ' of gene).

\section{Spotting assays}

The URA3 reporter expression spotting assays were performed in two biological replicates as follows. Freshly grown single colony isolates of each strain were grown in liquid YPD media to mid log phase Cells were subsequently collected, re-suspended in sterile water, and normalized to an $\mathrm{OD}_{600}=1$ (approximately $3 \times 10^{7}$ cells $/ \mathrm{ml}$ ). The normalized cell suspensions were subjected to 10 -fold serial dilutions and $4 \mu \mathrm{l}$ of each dilution was spotted onto standard SD- complete media, SD media without uracil, and SD media with 5-FOA at a final concentration of $1000 \mathrm{mg} / \mathrm{L}$ and uracil at a final concentration of $50 \mathrm{mg} / \mathrm{L}$. Plates were incubated at $30^{\circ} \mathrm{C}$ and growth was analyzed after 48 hours.

\section{rDNA Reporter Propagation Assays}

The URA+ status of each reporter containing strain was first confirmed by growth on SD media lacking uracil. Saturated overnights were then prepared from single colony isolates of each confirmed strain in liquid YPD media. Cultures were prepared from the overnights in 50ml YPD media and grown at $30^{\circ} \mathrm{C}$ to mid log phase. Cells were subsequently collected, washed once, resuspended in sterile deionized water, and normalized to an $\mathrm{OD}_{600}=0.5$. Normalized cell suspensions were subsequently diluted 10 -fold and $250 \mu \mathrm{l}$ of each dilution was plated on $25 \mathrm{ml}$ SD 5-FoA plates. Plates were incubated at $30^{\circ} \mathrm{C}$ for 16 hours. A total of 96 well-isolated colonies were randomly picked from each 5-FoA plate using the Genetix QPix-2 colony picking robot and deposited onto non-selective solid YPD plates. Plates were incubated for 5 days at $30^{\circ} \mathrm{C}$. All 96 colonies on each YPD plate were then replica plated onto SD complete control media and SD media lacking uracil and incubated for 5 days at $30^{\circ} \mathrm{C}$ before being imaged.

\section{The authors declare they have no conflict of interest.}




\section{References}

Basson ME, Thorsness M \& Rine J (1986) Saccharomyces cerevisiae contains two functional genes encoding 3-hydroxy-3-methylglutaryl-coenzyme A reductase. Proc. Natl. Acad. Sci. U. S. A. 83: 5563-5567

Bentley-DeSousa A, Holinier C, Moteshareie H, Tseng YC, Kajjo S, Nwosu C, Amodeo GF, BondyCandidate Targets of Lysine Polyphosphorylation Uncovers a Conserved Network Implicated in Ribosome Biogenesis. Cell Rep. 22: 3427-3439

Benton BM, Zang JH \& Thorner J (1994) A novel FK506- and rapamycin-binding protein (FPR3 gene product) in the yeast Saccharomyces cerevisiae is a proline rotamase localized to the nucleolus. $J$. Cell Biol. 127: 623-639

Collins SR, Miller KM, Maas NL, Roguev A, Fillingham J, Chu CS, Schuldiner M, Gebbia M, Recht J,

Costanzo M, Baryshnikova A, Bellay J, Kim Y, Spear ED, Sevier CS, Ding H, Koh JLY, Toufighi K, Mostafavi S, Prinz J, St Onge RP, VanderSluis B, Makhnevych T, Vizeacoumar FJ, Alizadeh S, Bahr S, Brost RL, Chen Y, Cokol M, et al (2010) The genetic landscape of a cell. Science 327: 425-31 Available at: http://www.ncbi.nlm.nih.gov/pubmed/24994677 J, Lee SD, Pelechano V, Styles EB, Billmann M, Van Leeuwen J, Van Dyk N, Lin ZY, Kuzmin E, Nelson J, Piotrowski JS, Srikumar T, et al (2016) A global genetic interaction network maps a wiring diagram of cellular function. Science (80-. ). 353:

Darlington CD \& Moffett AA (1930) Primary and secondary chromosome balance in Pyrus. J. Genet. 22: 129-151 Available at: https://doi.org/10.1007/BF02983843

Davey M, Hannam C, Wong C \& Brandl CJ (2000) The yeast peptidyl proline isomerases FPR3 and FPR4, in high copy numbers, suppress defects resulting from the absence of the E3 ubiquitin ligase TOM1. Mol. Gen. Genet. MGG 263: 520-526 Available at: 
Dilworth D, Upadhyay SK, Bonnafous P, Edoo AB, Bourbigot S, Pesek-Jardim F, Gudavicius G, Serpa JJ, Petrotchenko E V., Borchers CH, Nelson CJ \& MacKereth CD (2017) The basic tilted helical bundle domain of the prolyl isomerase FKBP25 is a novel double-stranded RNA binding module. Nucleic Acids Res. 45: 1-16

Dolinski K, Muir S, Cardenas M \& Heitman J (1997) All cyclophilins and FK506 binding proteins are, individually and collectively, dispensable for viability in Saccharomyces cerevisiae. Proc. Natl. Acad. Sci. U. S. A. 94: 13093-13098 Available at: http:/www.pubmedcentral.nih.gov/articlerender.fcgi?artid=24268\&tool=pmcentrez\&rendertype= abstract

Dutta S, Akey I V., Dingwall C, Hartman KL, Laue T, Nolte RT, Head JF \& Akey CW (2001) The crystal structure of nucleoplasmin-core: Implications for histone binding and nucleosome assembly. Mol. Cell 8: 841-853

Edlich-Muth C, Artero JB, Callow P, Przewloka MR, Watson AA, Zhang W, Glover DM, Debski J, Dadlez M, Round AR, Forsyth VT \& Laue ED (2015) The pentameric nucleoplasmin fold is present in Drosophila FKBP39 and a large number of chromatin-related proteins. J. Mol. Biol. 427: 1949-1963 Available at: http://dx.doi.org/10.1016/j.jmb.2015.03.010

Ellahi A, Thurtle DM \& Rine J (2015) The chromatin and transcriptional landscape of native saccharomyces cerevisiae telomeres and subtelomeric domains. Genetics 200: 505-521

Fermi B, Bosio MC \& Dieci G (2016) Promoter architecture and transcriptional regulation of Abf1dependent ribosomal protein genes in Saccharomyces cerevisiae. Nucleic Acids Res. 44: 61136126

Force A, Lynch M, Pickett FB, Amores A, Yan YL \& Postlethwait J (1999) Preservation of duplicate genes by complementary, degenerative mutations. Genetics 151: 1531-45 Available at: http://www.ncbi.nlm.nih.gov/pubmed/10101175

Francino MP (2005) An adaptive radiation model for the origin of new gene functions. Nat. Genet. 37: 573-577

Ghosh A \& Cannon JF (2013) Analysis of Protein Phosphatase-1 and Aurora Protein Kinase Suppressors Reveals New Aspects of Regulatory Protein Function in Saccharomyces cerevisiae.

\section{PLoS One 8:}


Gottlieb S \& Esposito RE (1989) A new role for a yeast transcriptional silencer gene, SIR2, in regulation of recombination in ribosomal DNA. Cell 56: 771-6 Available at: http://www.ncbi.nlm.nih.gov/pubmed/2647300

Houseley J \& Tollervey D (2008) The nuclear RNA surveillance machinery: The link between ncRNAs and genome structure in budding yeast? Biochim. Biophys. Acta - Gene Regul. Mech. 1779: 23946 Available at: http://www.ncbi.nlm.nih.gov/pubmed/18211833 [Accessed October 6, 2014]

Hughes AL (1994) The evolution of functionally novel proteins after gene duplication. Proc. R. Soc. London. Ser. B Biol. Sci. 256: 119-124 Available at: http://rspb.royalsocietypublishing.org/lookup/doi/10.1098/rspb.1994.0058

Innan H \& Kondrashov F (2010) The evolution of gene duplications: Classifying and distinguishing

Huh W-K, Falvo J V, Gerke LC, Carroll AS, Howson RW, Weissman JS \& O'Shea EK (2003) Global analysis of protein localization in budding yeast. Nature 425: 686-91 Available at: http://www.ncbi.nlm.nih.gov/pubmed/14562095 between models. Nat. Rev. Genet. 11: 97-108

Johnston M, Hillier L, Riles L, Albermann K, André B, Ansorge W, Benes V, Brückner M, Delius H, Dubois E, Düsterhöft A, Entian KD, Floeth M, Goffeau A, Hebling U, Heumann K, Heuss-Neitzel D, Hilbert H, Hilger F, Kleine K, et al (1997) The nucleotide sequence of Saccharomyces cerevisiae chromosome XII. Nature 387: 87-90 Available at: http://www.ncbi.nlm.nih.gov/pubmed/9169871

Karyn Schmidt and J. Scott Butler (2013) Nuclear RNA Surveillance: Role of TRAMP in Controlling Exosome Specificity. Wiley Interdiscip Rev 4: 217-231

Kataoka T, Powers S, McGill C, Fasano O, Strathern J, Broach J \& Wigler M (1984) Genetic analysis of yeast RAS1 and RAS2 genes. Cell 37: 437-45 Available at: 
http://www.ncbi.nlm.nih.gov/pubmed/6327067

Kellis M, Birren BW \& Lander ES (2004) Proof and evolutionary analysis of ancient genome duplication in the yeast Saccharomyces cerevisiae. Nature 428: 617-624

Kobayashi T, Horiuchi T, Tongaonkar P, Vu L \& Nomura M (2004) SIR2 regulates recombination between different rDNA repeats, but not recombination within individual rRNA genes in yeast. Cell 117: 441-453

Krogan NJ, Cagney G, Yu H, Zhong G, Guo X, Ignatchenko A, Li J, Pu S, Datta N, Tikuisis AP, 546

Koztowska M, Tarczewska A, Jakób M, Bystranowska D, Taube M, Kozak M, Czarnocki-Cieciura M, Dziembowski A, Ort Owski M, Tkocz K \& Ozyhar A (2017) Nucleoplasmin-like domain of FKBP39 from Drosophila melanogaster forms a tetramer with partly disordered tentacle-like Cterminal segments. Sci. Rep. 7: 1-14

Kuzuhara T \& Horikoshi M (2004) A nuclear FK506-binding protein is a histone chaperone regulating rDNA silencing. Nat. Struct. Mol. Biol. 11: 275-83 Available at: http://www.ncbi.nlm.nih.gov/pubmed/14981505 [Accessed April 29, 2014]

LaCava J, Houseley J, Saveanu C, Petfalski E, Thompson E, Jacquier A \& Tollervey D (2005) RNA degradation by the exosome is promoted by a nuclear polyadenylation complex. Cell 121: 713-24 Available at: http://www.ncbi.nlm.nih.gov/pubmed/15935758 [Accessed July 28, 2014]

Van Leeuwen F, Gafken PR \& Gottschling DE (2002) Dot1p modulates silencing in yeast by methylation of the nucleosome core. Cell 109: 745-756

van Leeuwen F \& Gottschling DE (2002) Assays for gene silencing in yeast. Methods Enzymol. 350: 165-86 Available at: http://www.ncbi.nlm.nih.gov/pubmed/12073311

Leung A, Jardim F-P, Savic N, Monneau YR, González-Romero R, Gudavicius G, Eirin-Lopez JM, Bartke T, Mackereth CD, Ausió J \& Nelson CJ (2017) Basic surface features of nuclear FKBPs facilitate chromatin binding. Sci. Rep. 7: 3795 Available at: http://www.nature.com/articles/s41598-017-04194-7

Li H \& Durbin R (2010) Fast and accurate short-read alignment with Burrows-Wheeler transform. 
Li H \& Luan S (2010) AtFKBP53 is a histone chaperone required for repression of ribosomal RNA gene expression in Arabidopsis. Cell Res. 20: 357-66 Available at: http://dx.doi.org/10.1038/cr.2010.22

Li M, Valsakumar V, Poorey K, Bekiranov S \& Smith JS (2013) Genome-wide analysis of functional sirtuin chromatin targets in yeast. Genome Biol. 14:

Macqueen AJ \& Roeder GS (2009) Fpr3 and Zip3 ensure that initiation of meiotic recombination precedes chromosome synapsis in budding yeast. Curr. Biol. 19: 1519-26 Available at: http://www.pubmedcentral.nih.gov/articlerender.fcgi?artid=2926792\&tool=pmcentrez\&rendertyp $\mathrm{e}=$ abstract [Accessed August 14, 2014]

Manning-Krieg UC, Henríquez R, Cammas F, Graff P, Gavériaux S \& Movva NR (1994) Purification of FKBP-70, a novel immunophilin from Saccharomyces cerevisiae, and cloning of its structural gene, FPR3. FEBS Lett. 352: 98-103 Available at: http://www.ncbi.nlm.nih.gov/pubmed/7925954

Milliman EJ, Yadav N, Chen YC, Muddukrishna B, Karunanithi S \& Yu MC (2012) Recruitment of Rpd3 to the Telomere Depends on the Protein Arginine Methyltransferase Hmt1. PLoS One 7: 111

Neef DW \& Kladde MP (2003) Polyphosphate Loss Promotes SNF/SWI- and Gen5-Dependent Mitotic Induction of PHO5. Mol. Cell. Biol. 23: 3788-3797

Nelson CJ, Santos-Rosa H \& Kouzarides T (2006) Proline isomerization of histone H3 regulates lysine methylation and gene expression. Cell 126: 905-16 Available at: http://www.ncbi.nlm.nih.gov/pubmed/16959570 [Accessed June 4, 2014]

Nomura M, Nogi Y, Oakes M \& Masayasu Nomura, Yasuhisa Nogi and MO (2004) Transcription of rDNA in the yeast Saccharomyces cerevisiae. Mol. Biol. Intell. ...: 1-25 Available at: http://www.landesbioscience.com/iu/Olson_9781587066221.pdf\#page=141 [Accessed May 1, 2014]

Ohkuni K, Abdulle R \& Kitagawa K (2014) Degradation of Centromeric Histone H3 Variant Cse4 Requires the Fpr3 Peptidyl-prolyl Cis-Trans Isomerase. Genetics 196: 1041-1045 Available at: http://www.ncbi.nlm.nih.gov/pubmed/24514906 [Accessed August 14, 2014]

Ohno S (1970) Part 3: Why Gene Duplication? In Evolution by Gene Duplication pp 59-88. SpringerVerlag 
Park S-K, Xiao H \& Lei M (2014) Nuclear FKBPs, Fpr3 and Fpr4 affect genome-wide genes transcription. Mol. Genet. Genomics 289: 125-36 Available at: http://www.ncbi.nlm.nih.gov/pubmed/24297734 [Accessed April 29, 2014]

Pemberton TJ (2006) Identification and comparative analysis of sixteen fungal peptidyl-prolyl cis/trans isomerase repertoires. BMC Genomics 7: 244 Available at: http://bmcgenomics.biomedcentral.com/articles/10.1186/1471-2164-7-244

Reis CC \& Campbell JL (2007) Contribution of Trf4/5 and the nuclear exosome to genome stability through regulation of histone mRNA levels in Saccharomyces cerevisiae. Genetics 175: 993-1010 Available at:

San Paolo S, Vanacova S, Schenk L, Scherrer T, Blank D, Keller W \& Gerber AP (2009) Distinct roles of non-canonical poly(A) polymerases in RNA metabolism. PLoS Genet. 5: 13-17

Schmitt ME, Brown TA \& Trumpower BL (1990) A rapid and simple method for preparation of RNA from Saccharomyces cerevisiae. Nucleic Acids Res. 18: 3091-3092

Shan X, Xue Z \& Mélèse T (1994) Yeast NPI46 encodes a novel prolyl cis-trans isomerase that is located in the nucleolus. J. Cell Biol. 126: 853-862 Available at:

Sinclair D a \& Guarente L (1997) Extrachromosomal rDNA circles--a cause of aging in yeast. Cell 91: http:/www.pubmedcentral.nih.gov/articlerender.fcgi?artid=2120118\&tool=pmcentrez\&rendertyp 1033-42 Available at: http://www.ncbi.nlm.nih.gov/pubmed/9428525

Stirling PC, Bloom MS, Solanki-Patil T, Smith S, Sipahimalani P, Li Z, Kofoed M, Ben-Aroya S, Myung K \& Hieter P (2011) The complete spectrum of yeast chromosome instability genes identifies candidate cin cancer genes and functional roles for astra complex components. PLoS Genet. 7: 9-13 Nop53p is a novel nucleolar $60 \mathrm{~S}$ ribosomal subunit biogenesis protein. Biochem. J 388: 819-826

Tarasov A, Vilella AJ, Cuppen E, Nijman IJ \& Prins P (2015) Sambamba: fast processing of NGS alignment formats. Bioinformatics 31: 2032-4 Available at: http://www.ncbi.nlm.nih.gov/pubmed/25697820 
Tong AHY \& Boone C (2006) Synthetic genetic array analysis in Saccharomyces cerevisiae. Methods Mol. Biol. (Totowa, N.J.) 313: 171-92 Available at: http://www.pubmedcentral.nih.gov/articlerender.fcgi?artid=3276145\&tool=pmcentrez\&rendertyp $\mathrm{e}=\mathrm{abstract}$

Utsugi T, Hirata A, Sekiguchi Y, Sasaki T, Toh-e A \& Kikuchi Y (1999) Yeast tom1 mutant exhibits pleiotropic defects in nuclear division, maintenance of nuclear structure and nucleocytoplasmic transport at high temperatures. Gene 234: 285-295 Available at: http://linkinghub.elsevier.com/retrieve/pii/S0378111999001973

Vogelauer M, Cioci F, Bordi L, Camilloni G, Molecolare B, La R \& Moro PA (2000) In vivo Studies of the Non-transcribed Spacer Region of rDNA in Saccharomyces cerevisiae. Food Technol. Biotechnol. 38: 315-321

Warner JR (1999) The economics of ribosome biosynthesis in yeast. Trends Biochem. Sci. 24: 437-40 Available at: http://www.ncbi.nlm.nih.gov/pubmed/10542411

Wery M, Ruidant S, Schillewaert S, Leporé N \& Lafontaine DLJ (2009) The nuclear poly(A) polymerase and Exosome cofactor Trf5 is recruited cotranscriptionally to nucleolar surveillance. RNA 15: 406-19 Available at: http://www.pubmedcentral.nih.gov/articlerender.fcgi?artid=2657017\&tool=pmcentrez\&rendertyp $\mathrm{e}=\mathrm{abstract}$

Wolfe KH \& Shields DC (1997) Molecular evidence for an ancient duplication of the entire yeast genome. Nature 387: 708-713

Wolin SL, Sim S \& Chen X (2012) Nuclear noncoding RNA surveillance: Is the end in sight? Trends Genet. 28: 306-313

Xiao H, Jackson V \& Lei M (2006) The FK506-binding protein, Fpr4, is an acidic histone chaperone. FEBS Lett. 580: 4357-64 Available at: http://www.ncbi.nlm.nih.gov/pubmed/16846601 [Accessed April 29, 2014]

Young BP \& Loewen CJR (2013) Balony: a software package for analysis of data generated by synthetic genetic array experiments. BMC Bioinformatics 14: 354 Available at: 
Figure 1 -Fpr3 and Fpr4 have separate, co-operative and redundant functions.

756 A. Domain architectures of Fpr3 and Fpr4. Both proteins have an N-terminal nucleoplasmin-like domain with characteristic patches of acidic and basic residues, and a C-terminal peptidyl prolyl isomerase domain.

B. Schematic illustrating modified paralog SGA workflow. Spores from a single cross of the double deletion $\Delta f p r 3 \Delta f p r 4$ query to the 4784 - strain DMA are manipulated to generate three separate sets of meiotic progeny for interactome analysis.

762

C. On top, Venn diagram illustrating numbers of synthetic sick and synthetic lethal genetic interactors unique to FPR3 and FPR4, and shared among both of them. On bottom, number of masked redundant synthetic sick and synthetic lethal genetic interactions only detectable in double deletion $\Delta f p r 3 \Delta f p r 4$ mutants.

D. Network illustrating complex related ontologies enriched among unique and shared genetic interactors of FPR3 and FPR4. Asterix denotes genetic interactions with the SWI/SNF component coding genes which were confirmed to be shared among Fpr3 and Fpr4 with spotting assays.

Figure 2 - The TRAMP5 nuclear RNA exosome is a masked genetic interactor of FPR3 and FPR4.

A. Mean colony size ratios of experimental $(\Delta f p r 3 \Delta f p r 4 \Delta x x x)$ triple mutants relative to control $\Delta x x x$ total haploid meiotic progeny for select redundant synthetic sick or lethal genetic interactors. Asterix indicates that $2 / 3$ replicates for the $\Delta f p r 3 \Delta f p r 4 \Delta r r p 6$ deletion mutant were below the synthetic sick/ lethal cut-off threshold. left). Complex components coded for by redundant genetic interactors of FPR3 or FPR4 are colored red. Pink text labels indicate components of complex coded for by essential genes. Illustration is adapted from (Wolin et al, 2012).

C. Growth curves depicting $\mathrm{OD}_{600}$ vs time for select triple deletion mutants and corresponding total haploid meiotic progeny control populations.

Figure 3 -Suppressor genetic interactions support chromatin-centric functions for Fpr3 and Fpr4. 
A. On top, Venn diagram illustrating numbers of suppressor interactors unique to FPR3 and FPR4 and shared among both of them. On bottom, number of masked redundant suppressor genetic interactions only detectable in double deletion $\Delta f p r 3 \Delta f p r 4$ mutants.

B. Plot of fitness ratios for all $\Delta f p r 3 \Delta f p r 4 \Delta x x x$ triple mutants relative to $\Delta x x x$ total haploid meiotic progeny controls. Green dots indicate all synthetic sick/ lethal genetic interactions, red dots indicate all suppressor genetic interactions. Threshold cut-offs are indicated by red and green dashed horizontal lines. Fitness ratios associated with genes coding for components of chromatin modifiers are labeled and accompanied with schematic illustrations of complex components coded for by the synthetic sick genetic interactors (illustrated in green boxes) and suppressor genetic interactors (illustrated in red boxes). Components coded for by interacting genes are colored. Components coded for by noninteracting genes are black and white. Red text illustrates components coded for by essential genes absent from the non-essential yeast DMA.

A. Scatter plots indicating the correlation of gene expression between wt and $\Delta f p r 3 \Delta f p r 4$ and wt and $\Delta$ sir2 deletion mutants.

B. Scatter plots indicating the correlation of gene expression between $\Delta f p r 3 \Delta \operatorname{trf} 5$ double mutants and $\Delta f p r 3 \Delta f p r 4 \Delta \operatorname{trf5}$ triple deletion mutants. mutants. Enriched genes were classified by molecular function, biological process, cellular component, and MIPS functional database classification by FunSpec (http://funspec.med.utoronto.ca/).

\section{Figure 5 - A signature of abortive transcription is present in $\Delta f p r 3 \Delta f p r 4$ yeast.}

A. Plot of total averaged upregulated, downregulated and unchanged transcripts generated form $\Delta f p r 3 \Delta \operatorname{trf5}$ double mutants (left) and $\Delta f p r 3 \Delta f p r 4 \Delta \operatorname{trf5}$ triple mutants (right).
D. Model illustrating Fpr4 building chromatin at gene promoters. (left), UTP9 (right).

\section{Read map illustrating an example of a non-differentially expressed gene without a signature of} abortive transcription IDP1.

\section{Figure 6 -Fpr3 and Fpr4 silence the non-transcribed spacers (NTS) of rDNA.}


317 A. Read maps illustrating transcripts generated from both strands of one of the tandem rDNA repeats in

$818 \Delta f p r 3 \Delta \operatorname{trf5}$ and $\Delta f p r 3 \Delta f p r 4 \Delta \operatorname{trf5}$ cells. Transcripts generated from the NTS2 locus are prezented in the

319 zoomed-in panel.

820 B. Ten-fold serial dilution spotting assays of single and double gene deletion mutants in strain

821 backgrounds carrying a URA3 reporter integrated either within NTS1 spacer of rDNA or at the $H M R a$

822 locus. Plates were grown on either standard defined complete media or on standard defined media

323 lacking uracil for 2 days at $37^{\circ} \mathrm{C}$.

$825 \quad$ Figure 7 - Fpr3 and Fpr4 are required for genomic stability at the rDNA locus.

326 A. Diagrams illustrating the propagation experiment carried out to assess frequency of reporter loss. In 827 a given population of cells, under non-selective conditions, URA3 may be in an accessible

828 euchromatin-like environment and therefore expressed (dark blue cells), in an inaccessible

829 heterochromatin-like environment and therefore silenced (light blue cells), or it may have been

830 permanently lost from the genome via recombination between repeats (orange cells).

831 B. Images of the 96 individuals selected for after propagation on SD-complete control media and on

832 SD- URA experimental media. Those growing on the experimental media represent the fraction of the

833 population in which the reporter was epigenetically silenced. Those that fail to grow indicate

834 permanent loss of the reporter.

835 C. Percentage of total colonies recovered after strain propagation that have retained or lost the ability

836 to grow on SD-complete media. 


\section{A Acidic Region Basic Region \\ Residue}

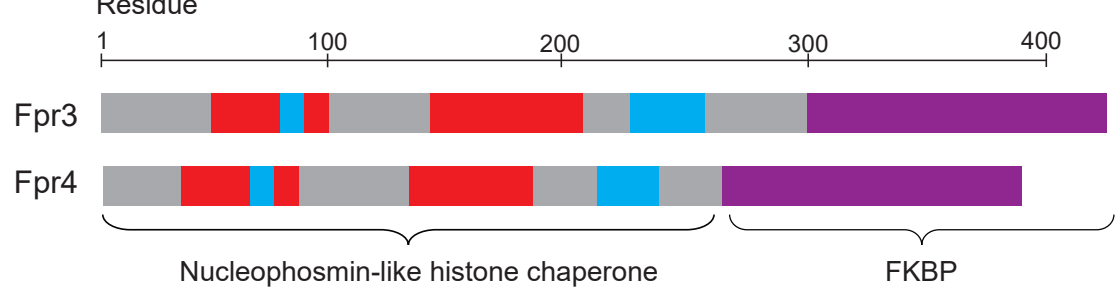

B meiotic progeny

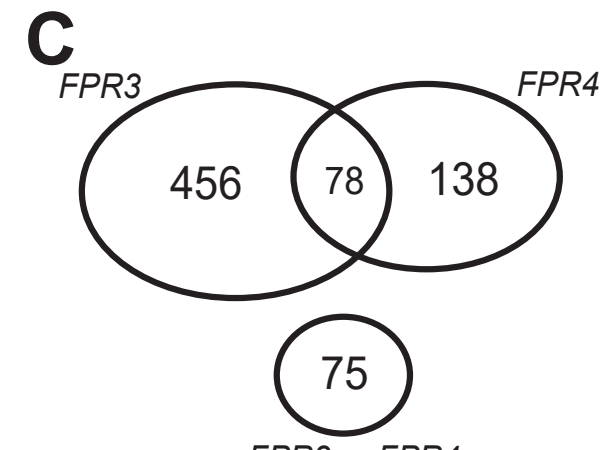

FPR3 or FPR4

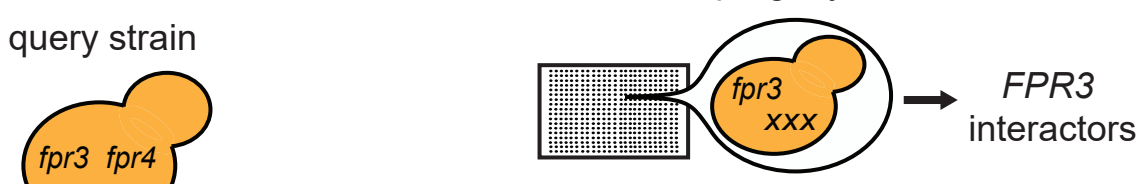

query strain
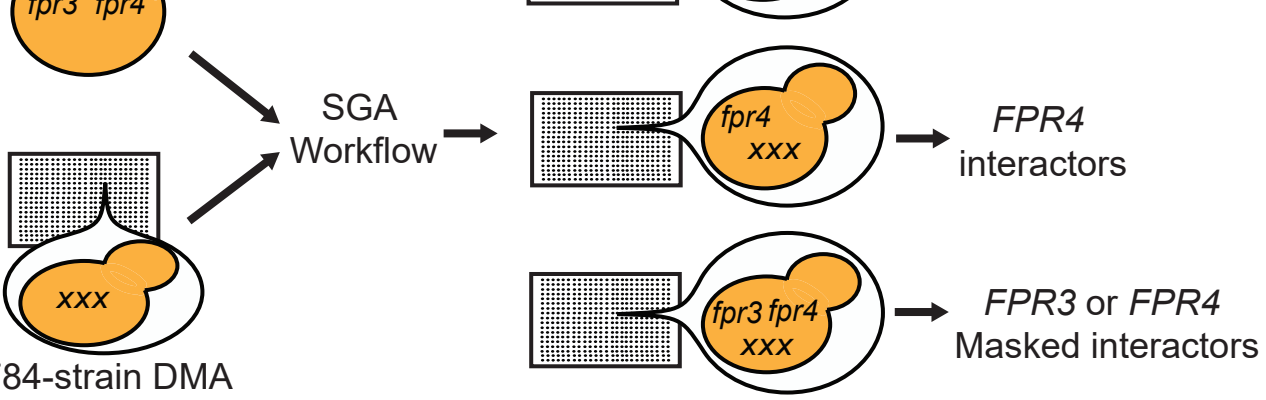

4784-strain DMA

D

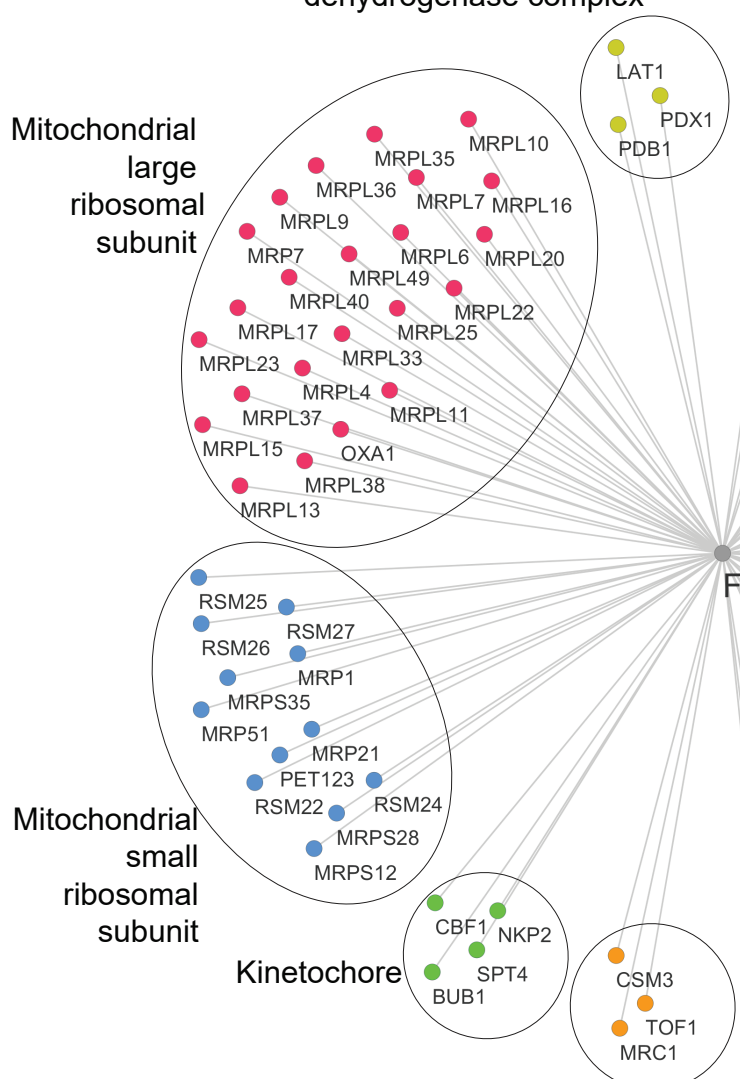

Tof1-Mrc1-Csm3 complex
Cytochrome bc1 complex

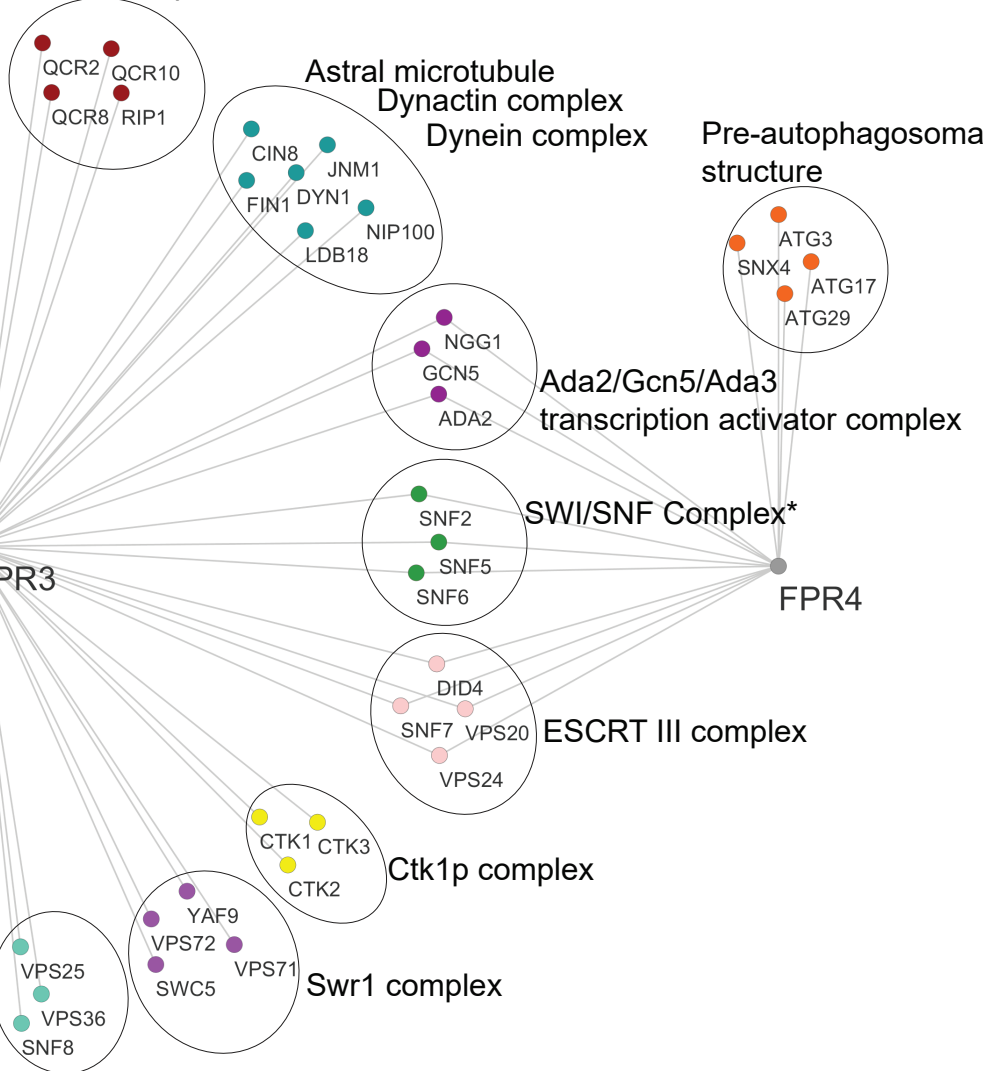

ESCRT II complex 
bioRxiv preprint doi: https://doi.org/10.1101/586347; this version posted March 24, 2019. The copyright holder for this preprint (which was not certified by peer review) is the author/funder. All rights reserved. No reuse allowed without permission.

A

GLO1

exp

glo1 fpr3fpr4glo1

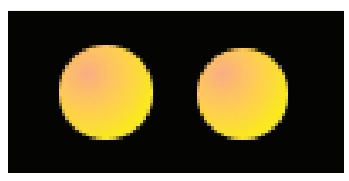

mean ratio: 1.0002

No interaction
TRF5

exp

trf5 fpr3fpr4trf5

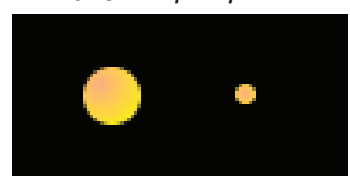

mean ratio: 0.1504

SS/SL interactor

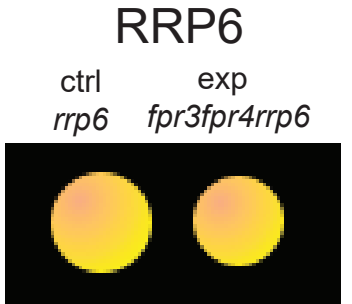

mean ratio: 0.8528

SS/SL interactor *
AIR1

$\begin{array}{cc}\text { ctrl } & \exp \\ \text { air1 } & \text { fpr3fpr4air1 }\end{array}$

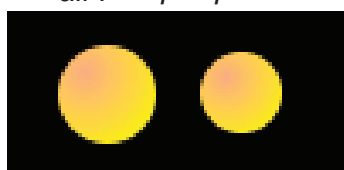

mean ratio: 0.6558

$\mathrm{SS} / \mathrm{SL}$ interactor

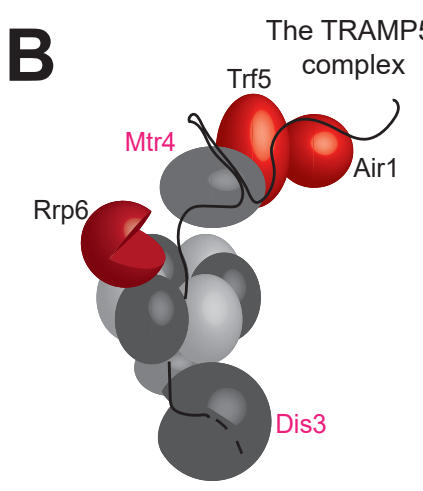

The Nuclear RNA Exosome

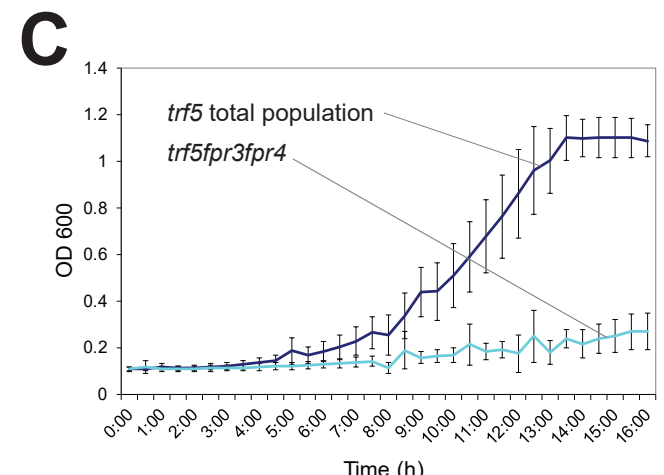

Time (h)

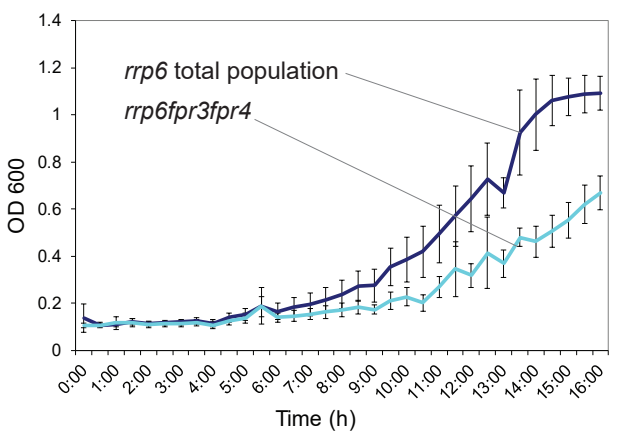


A

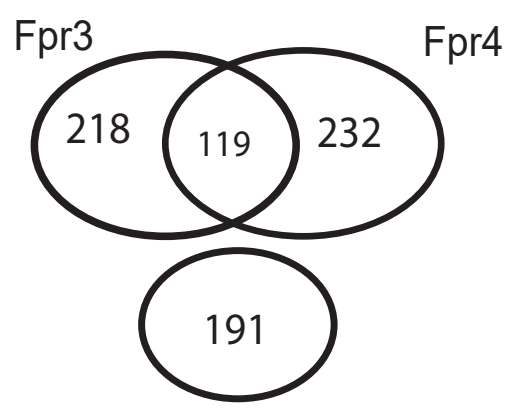

Fpr3 or Fpr4

Red text= essential gene,

**= gene not present in our DMA,

* gene is a masked interactor only

\section{The SWI/SNF Complex}

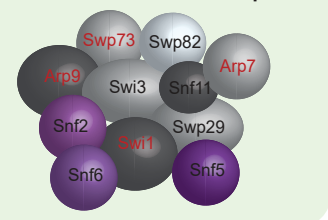

The ADA Complex

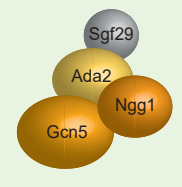

B
NAD+ dependent histone deacetylases

Hos2

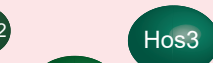
Hda1
HIR Complex

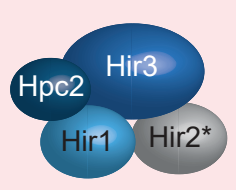

Set $1 /$ COMPASS Complex

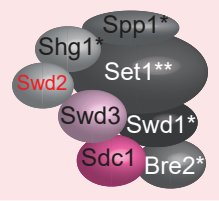

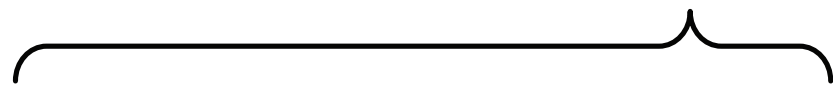

HOS2 HIR1 HDA1 HOS3 HPC2 SWD3 HIR3 SDC1

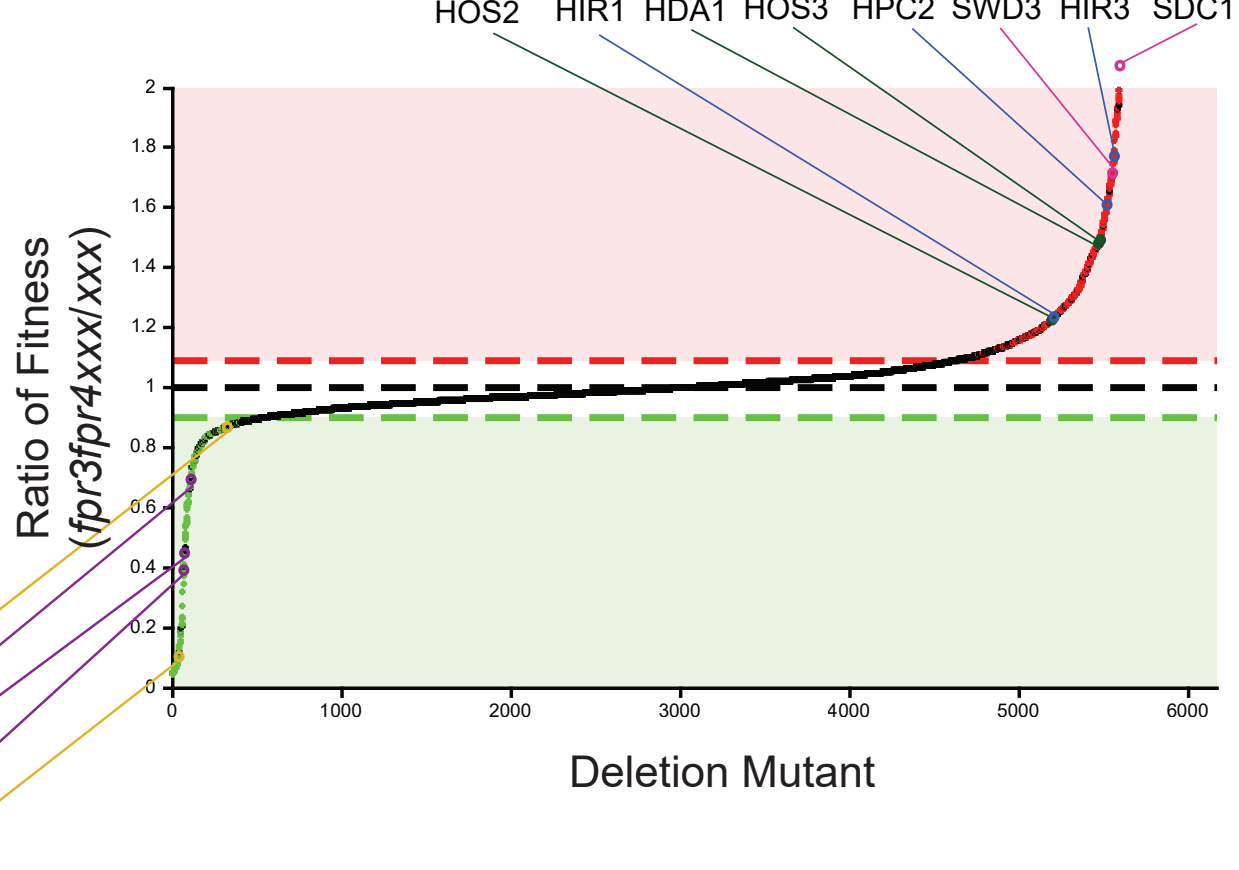


bioRxiv preprint doi: https://doi.org/10.1101/586347; this version posted March 24,2019 . The copyright holder for this preprint (which was not certified by peer review) is the author/funder. All rights reserved. No reuse allowed without permission.

A
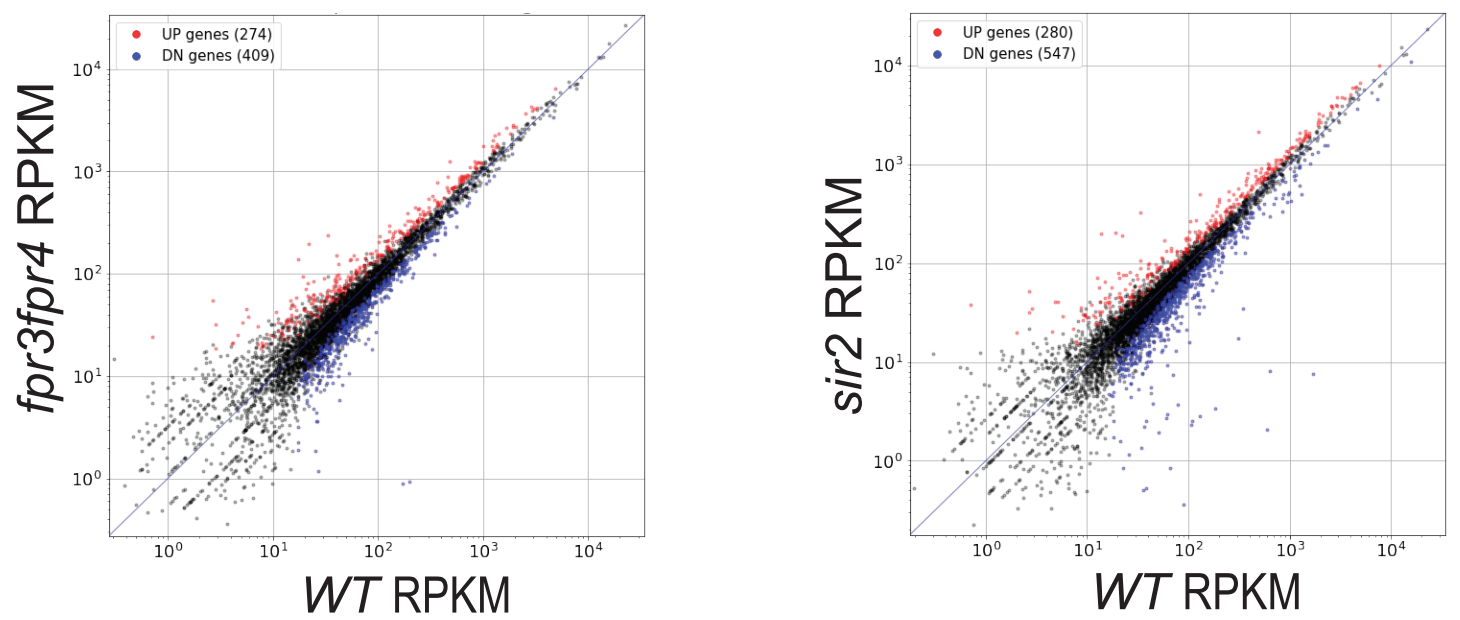

B
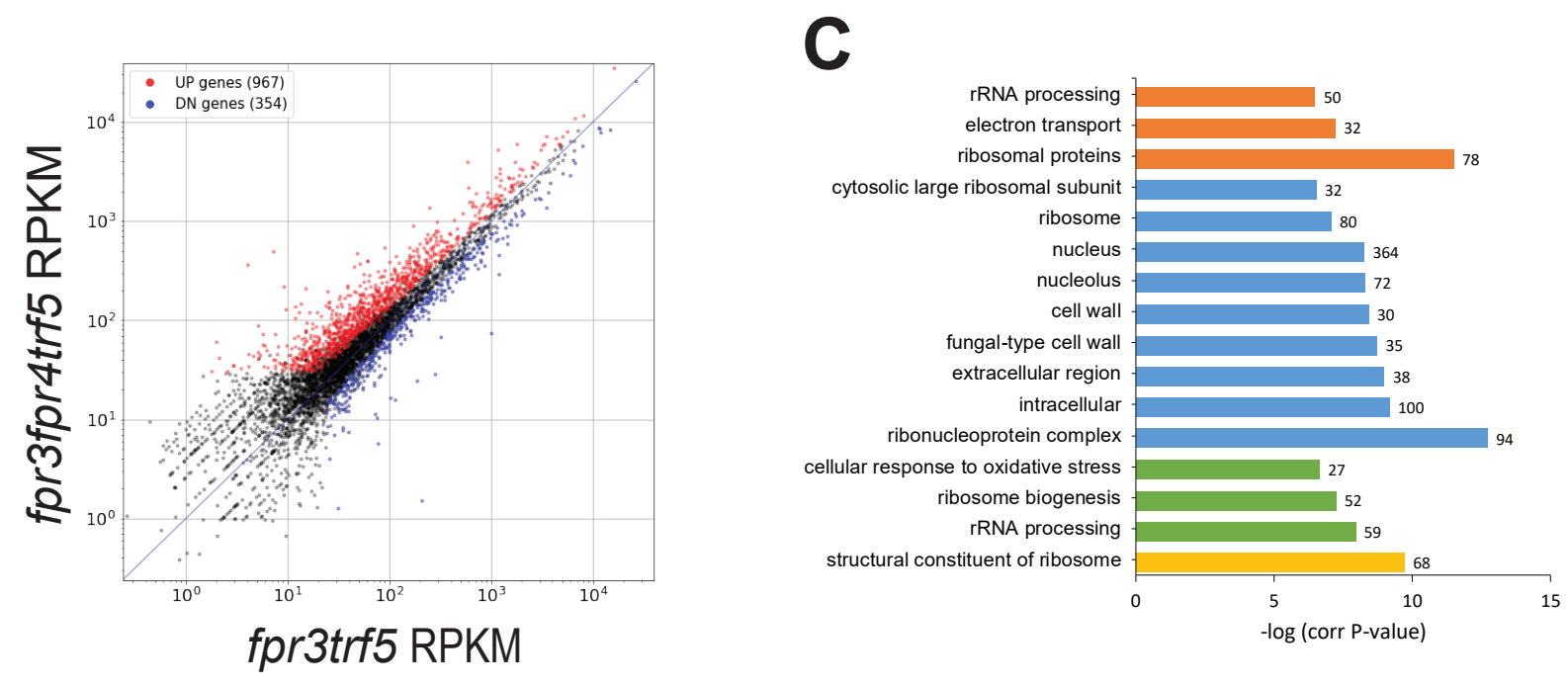

MIPS Functional Classification Cellular Component

Biological Process Molecular Function 
bioRxiv preprint doi: https://doi.org/10.1101/586347; this version posted March 24, 2019. The copyright holder for this preprint (which was not certified by peer review) is the author/funder. All rights reserved. No reuse allowed without permission.

A
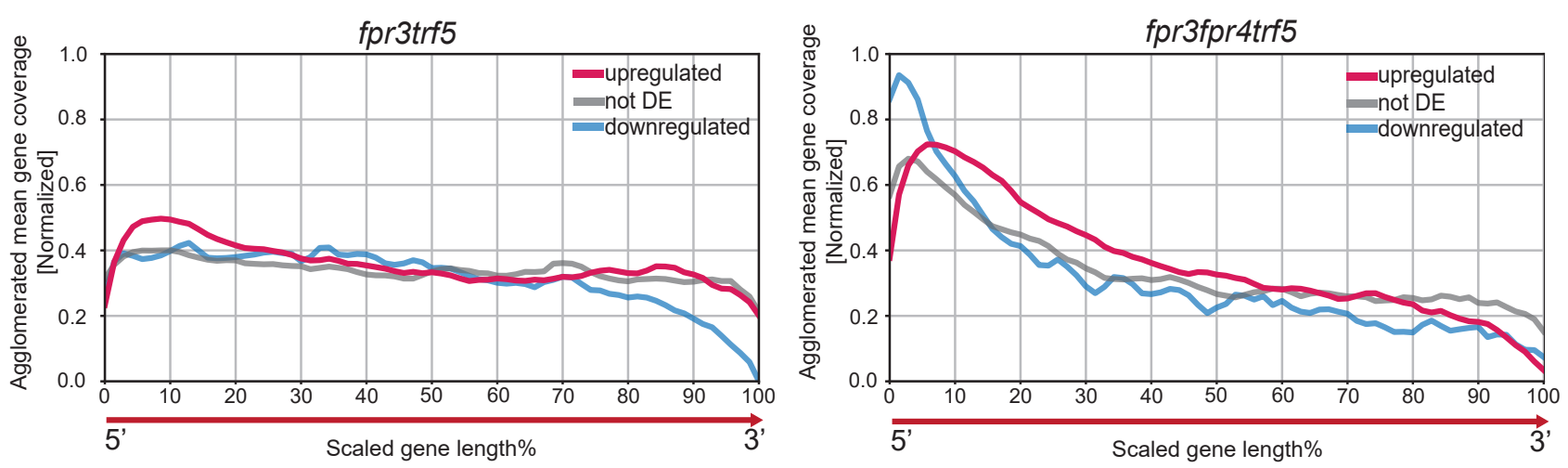

B
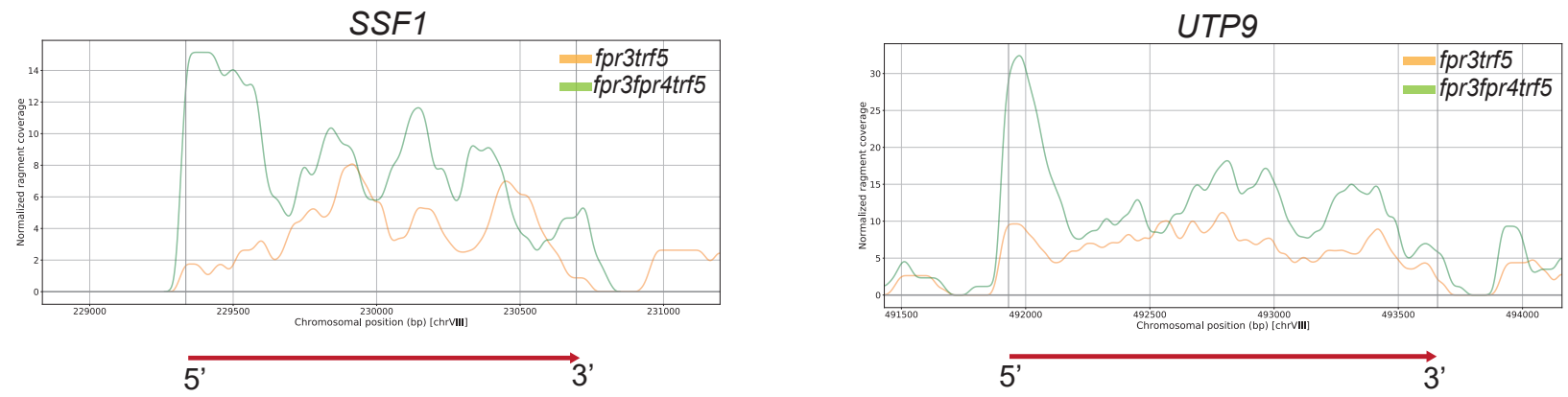

C

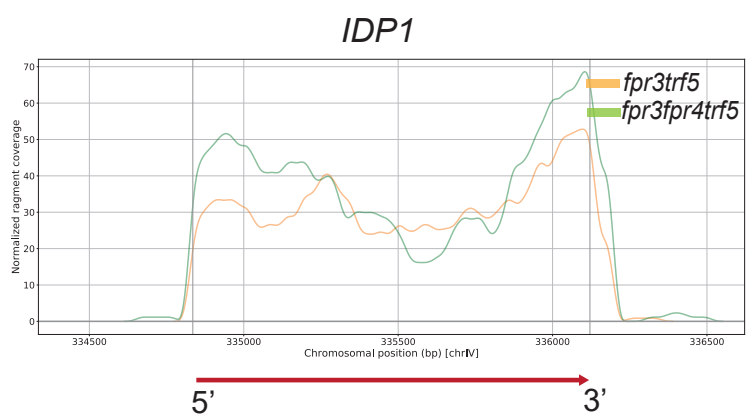

D tospospents

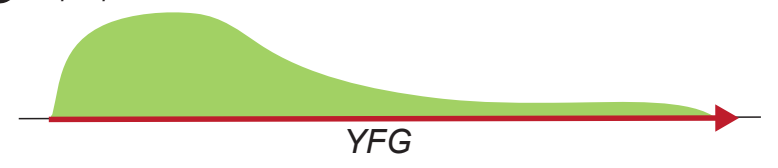

fpr3trf5
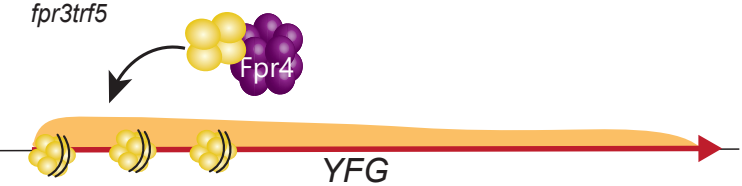
bioRxiv preprint doi: https://doi.org/10.1101/586347; this version posted March 24, 2019. The copyright holder for this preprint (which was not certified by peer review) is the author/funder. All rights reserved. No reuse allowed without permission.

A
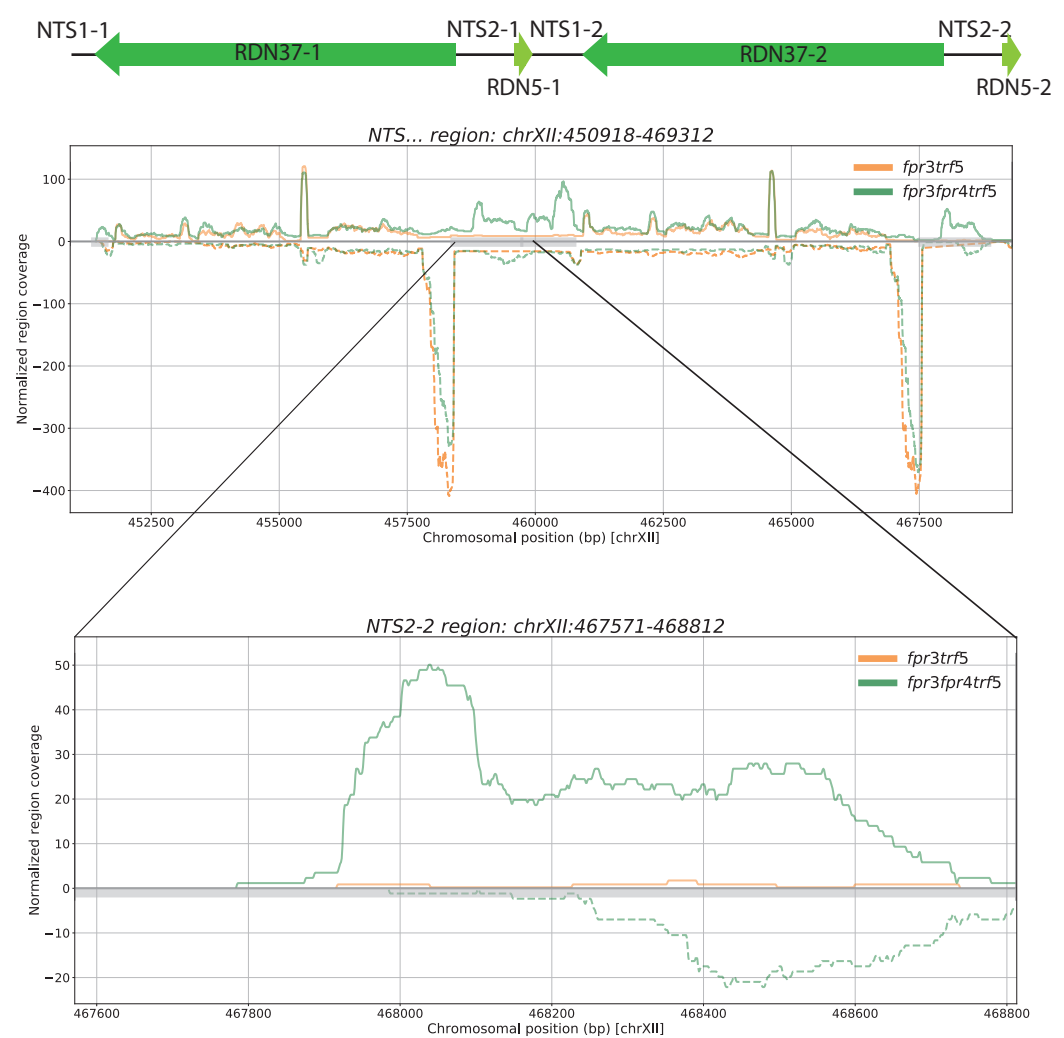

B
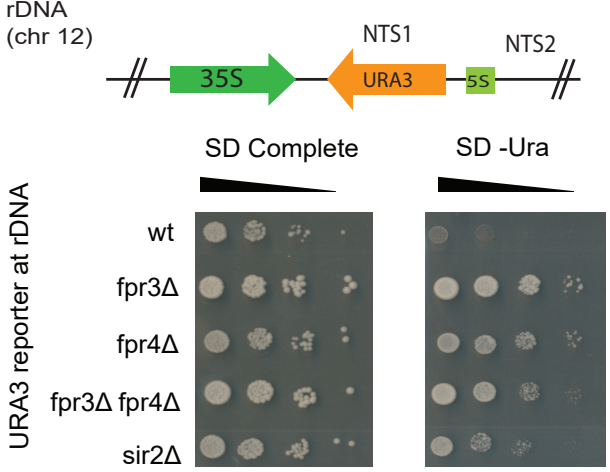

HML $\alpha$

(chr 3)

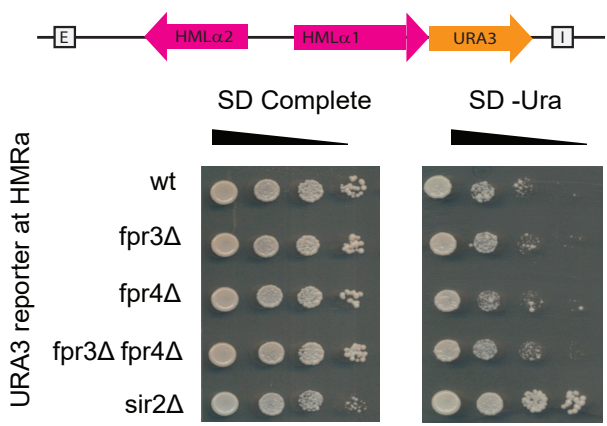


bioRxiv preprint doi: https://doi.org/10.1101/586347; this version posted March 24,2019 . The copyright holder for this preprint (which was not certified by peer review) is the author/funder. All rights reserved. No reuse allowed without permission.

A

Reporter in euchromatin
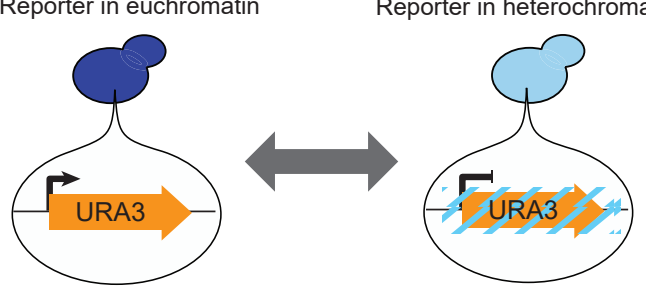

Reporter lost from genome

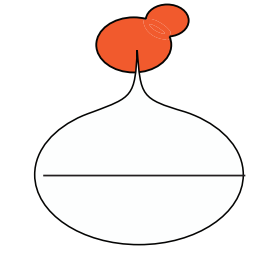

SD -URA
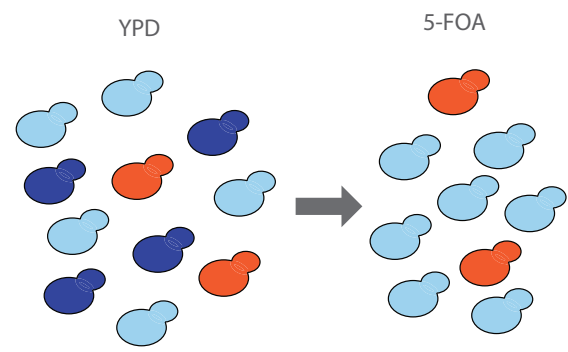

Propagate in non-selective environment

mixed population develops

Genotype: URA+/- mix

Phenotype: URA +/- mix
Enrich population for URAGenotype: URA+/- mix Phenotype: URA- mix

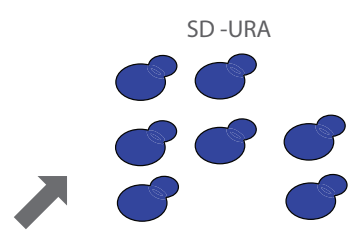

SD -complete
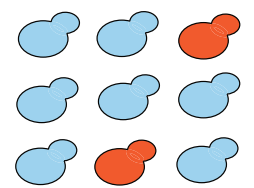

Isolate individuals and screen for heterochromatin silencing vs permanent reporter loss

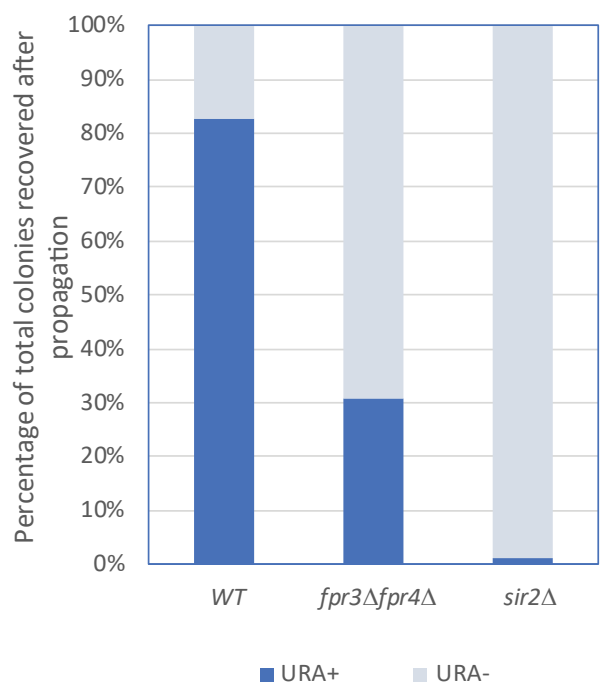

B

\section{SD complete SD-URA}

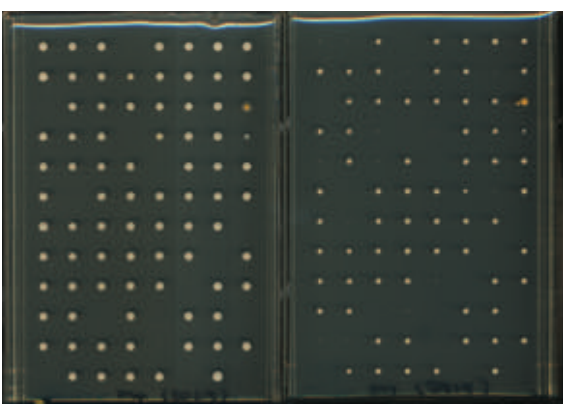

fpr3 3 fpr $4 \Delta$ SD complete SD-URA

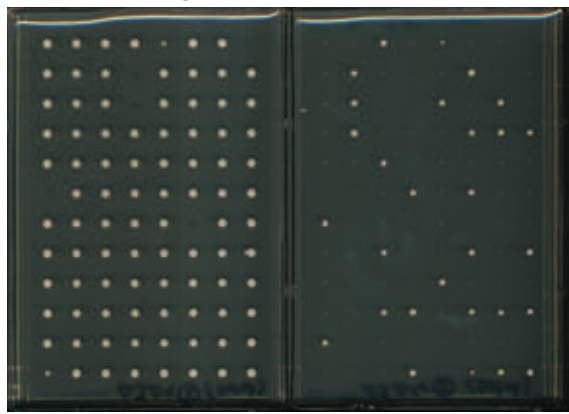

C

\section{SD complete SD -URA}

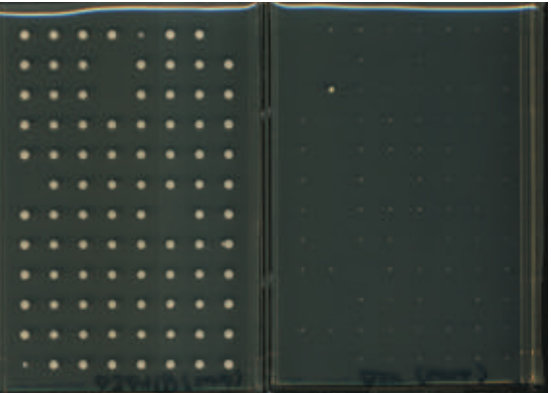

